\title{
Single-cell evaluation reveals innate immune arrested and lymphocytes shifting that promote development of preinvasive lung adenocarcinoma
}

\section{Chao Zhang}

Guangdong Lung Cancer Institute

\section{Alexandre Reuben}

The University of Texas MD Anderson Cancer Center https://orcid.org/0000-0003-4510-0382

Meredith Frank

MD Anderson Cancer Center

Si-Yang Liu

Guangdong Lung Cancer Institute

Zhi Xie

Guangdong Lung Cancer Institute

Li-Xu Yan

Guangdong Provincial People's Hospital

Zhen-Bin Qiu

Guangdong Lung Cancer Institute

Hui-Zhao Hong

Guangdong Lung Cancer Institute

Jian Su

Guangdong Lung Cancer Institute

Hai-Yan Tu

Guangdong Lung Cancer Institute

Xue-Ning Yang

Guangdong Lung Cancer Institute

Xu-Chao Zhang

Guangdong Lung Cancer Institute

Jing-Ji Yang

Guangdong Lung Cancer Institute

Qing Zhou

Guangdong Lung Cancer Institute

Yi-Long Wu

Guangdong Lung Cancer Institute

Jianjun Zhang 
The University of Texas MD Anderson Cancer Center https://orcid.org/0000-0001-7872-3477 Wen-zhao Zhong ( $\square$ syzhongwenzhao@scut.edu.cn )

Guangdong Lung Cancer Institute

\section{Genetics Article}

Keywords: preinvasive lung adenocarcinoma, multi-omics, single-cell sequencing, intercellular 18 heterogeneity, immune microenvironment

Posted Date: February 8th, 2022

DOI: https://doi.org/10.21203/rs.3.rs-1084110/v1

License: (9) This work is licensed under a Creative Commons Attribution 4.0 International License. Read Full License 


\section{1}

$$
2
$$

\title{
Original Article
}

\section{Title}

Single-cell evaluation reveals innate immune arrested and lymphocytes shifting that promote development of preinvasive lung adenocarcinoma

\section{Authors}

Chao Zhang ${ }^{1,5}$, Alexandre Reuben ${ }^{2,5}$, Meredith L. Frank ${ }^{2}$, Si-Yang Liu ${ }^{1}$, Zhi Xie ${ }^{1}$, Li-Xu Yan ${ }^{3}$, Zhen-

Bin Qiu ${ }^{1}$, Hui-Zhao Hong ${ }^{1}$, Jian Su${ }^{1}$, Hai-Yan Tu${ }^{1}$, Xue-Ning Yang ${ }^{1}$, Xu-Chao Zhang ${ }^{1}$, Jing-Ji Yang ${ }^{1}$, Qing Zhou ${ }^{1}$, Yi-Long Wu ${ }^{1}$, Jianjun Zhang ${ }^{3,4, *}$, Wen-Zhao Zhong ${ }^{1,6, *}$

\author{
Affiliation \\ ${ }^{1}$ Guangdong Lung Cancer Institute, Guangdong Provincial Key Laboratory of Translational Medicine in \\ Lung Cancer, Guangdong Provincial People's Hospital \& Guangdong Academy of Medical Sciences, \\ Guangzhou 510080, China; \\ ${ }^{2}$ Department of Thoracic/Head and Neck Medical Oncology, The University of Texas MD Anderson \\ Cancer Center, Houston, TX, 77030, USA. \\ ${ }^{3}$ Department of Pathology, Guangdong Provincial People's Hospital \& Guangdong Academy of Medical \\ Sciences, Guangzhou 510080, China; \\ ${ }^{4}$ Department of Genomic Medicine, The University of Texas MD Anderson Cancer Center, Houston, TX, \\ 77030, USA. \\ ${ }^{5}$ Authors contributed equally \\ ${ }^{6}$ Lead contact
}

*Correspondent author: Wen-Zhao Zhong, syzhongwenzhao@scut.edu.cn; Guangdong Lung Cancer Institute, Guangdong Provincial People's Hospital \& Guangdong Academy of Medical Sciences, Guangzhou, Guangdong 510080, P. R. China. Tel: 86-20-83877855, Fax: 86-20-83844620.

Jianjun Zhang, jzhang20@mdanderson.org; Department of Thoracic/Head and Neck Medical Oncology, The University of Texas MD Anderson Cancer Center, Houston, TX, 77030, USA.

\section{Competing interests}

Wen-Zhao Zhong received speech honoraria from AstraZeneca, Roche, Eli Lilly and Pfizer outside the submitted work. Yi-Long Wu received research funding from Roche and speech honoraria from AstraZeneca, Roche, Eli Lilly, Pfizer and Sanofi outside the submitted work, and he was a research consultant for AstraZeneca. Jian-Jun Zhang received personal fees from BMS, AstraZeneca, Geneplus, OrigMed, Innovent, grant from Merck, outside the submitted work; Other authors indicated no financial relationships.

\section{Word counts for body: 5642}

Number of Tables/Figures: 24 (6 figures and 1 supplementary table; 16 Supplementary figures) 
1 Summary

2 Cancer immunotherapy has demonstrated promising efficacy in the neoadjuvant setting for treating early-

3 stage non-small cell lung cancer (NSCLC). However, immunotherapy for preinvasive or early invasive

4 disease has been largely overlooked. We performed single-cell RNA and TCR sequencing for 266,804

5 high quality cells as well as whole exome sequencing (WES) and multiplex immunofluorescence (mIF)

6 on 31 pathologically confirmed preinvasive or early invasive lung adenocarcinoma (LUAD) with paired

7 adjacent tissues. We identified CD47-SIRPA as a key mediator of arresting innate immune response in

8 the tumor microenvironment of preinvasive LUAD. Additionally, highly intercellular heterogeneity was

9 observed and overexpression of KRT18 and PHLDA2 may promote malignant progression during

10 carcinogenesis. By integrating network analysis among tumor and immune cells, we established

11 comprehensive interactive landscape of preinvasive and early invasive LUAD, determining an imbalance

12 of Th17/Treg and increased LAMP $3^{+}$DCs may boost early tumor development. Our study provides

13 comprehensive genomic and immune features of preinvasive LUAD, highlighting novel therapeutic

14 targets which shed light on understanding how tumor microenvironment shapes neoplastic progression

15 during early evolution of LUAD.

17 Key words: preinvasive lung adenocarcinoma; multi-omics; single-cell sequencing; intercellular heterogeneity; immune microenvironment. 


\section{Introduction}

2 Despite of rapid developments in targeted therapies and immunotherapies $(1,2)$, prognosis of advanced lung cancer remains unsatisfactory $(3,4)$. Low-dose CT (LDCT)-guided lung cancer screening has significantly decreased lung cancer specific mortality by identification of earlier stage of lung cancer (5). Beyond surgical resection, the standard of care for early-stage lung cancer, prevention could be a promising strategy to reduce lung cancer mortality, particular for patients with multifocal diseases that were frequently detected in lung cancer screening trials. Lung cancer is a heterogenous disease (6-8) with distinct intrinsic molecular features and differential microenvironment between different patients and even within the same tumors. Such heterogenous nature could evolve as tumor progresses leading to inferior therapeutic efficacy and therapeutic dilemma in advanced lung cancer(9-11). Hence, understanding how cancer initiates and interacts with the microenvironment at its early phase of carcinogenesis may pave the way for personalized immunotherapy and other novel treatment options. Precursor lung adenocarcinoma known as atypical adenomatous hyperplasia (AAH), adenocarcinoma in situ (AIS) and minimally invasive adenocarcinoma (MIA) (12) has emerged increasingly due to widespread implementation of LDCT-guided lung cancer screening. All these lesions were clinically presented as subsolid nodules (SSNs) bearing relatively indolent tumor biology $(13,14)$. Although only $10 \%-20 \%$ of them may grow significantly within few years (15-17), the prognosis would be impaired once it progressed and all remaining multiple lesions including these precursor lung adenocarcinomas could be latent bombs. Therefore, exploring potential early interception targets might play essential role in restraining tumor development at its early stages and hopefully, provide noninvasive curative strategy. Recent studies from our group and others have described the molecular and immune evolution of LUAD precursors (18-22), but these analyses were at bulk level, which lacked the high-resolution to depict the complex interaction between evolving cancer cells and the microenvironment at a granular level. Advances in single-cell sequencing technology throughout these years have allowed us to study the tumor microenvironment in a more microcosmic manner and characterize the intricate immune landscape at a cellular level (23-25). A recent study evaluated microenvironment of LUAD manifested as pulmonary SSN and provided inspiring insight of indolent nature of SSN(26). However, no subsequent comparison of different histological states of LUAD precursors has been performed and the molecular events driving initiation and early progression of LUAD remain to be determined.

Herein, we conducted a multi-omics analysis including single-cell RNA transcriptome, single-cell T cell receptor (scTCR), whole exome sequencing (WES), and multiplex immunohistochemistry (mIHC) to decipher the single cell atlas among AIS, MIA and IAC as well as the mechanisms of diverse immune cells during precursor lesions 


\section{$3 \quad$ Results}

\section{Genomic features and cellular construction of early lung adenocarcinomas}

554 surgically resected sub-solid nodules pathologically diagnosed as lung adenocarcinoma (LUAD) derived from Asian patients were collected and histological subtypes were determined by Hematoxylin-eosin (HE) and thyroid transcription factor-1 (TTF-1) staining. Adenocarcinoma in situ (AIS) subtype was defined as 100\% lepidic patterns without invasive components. Minimally invasive adenocarcinoma (MIA) subtype was defined as lepidic predominant patterns with invasive component less than $0.5 \mathrm{~cm}$. Invasive adenocarcinoma (IAC) subtype was defined as mixed pathological patterns with tumor involvement through basement membrane. Study scheme, including filtering criteria for cells included in subsequent analysis, and overview of scRNA features were summarized (Figure 1a-d). scRNA sequencing revealed differences in infiltrating immune cell populations between adjacent lung tissue and tumor tissue histological subtypes (Figure 1e). Tumor ploidy as well as purity and clonal heterogeneity were assessed through FACETS (Supplementary Figure 2a) (27). Tumor mutation burden (TMB) and mutational landscape, including focal and chromosomal-level alterations, were presented in Supplementary Figure 2a. Among samples that underwent whole exome sequencing (WES), approximately 7-399 (median 28) nonsilent mutations were identified per case. Regardless of histological subtype, mutations in epidermal growth factor receptor (EGFR) gene were most chromosomal mutation common across patients (44\%) (Supplementary Figure 2a). Comparing across histological subtypes revealed that AIS/MIA subtypes carried higher incidences of mutations in BRAF and ERBB2 compared to early-stage LUAD, however, these mutations occurred exclusively of each other (Supplementary Figure 2a-b). Among the IAC subtype, driver mutations in EGFR and ERBB2 were found to occur exclusively of each other (Supplementary 2c). Similar to previous results (22, 28), significantly high bases transition compared to bases transversion was observed in precursor LUAD $(\mathrm{p}=0.012)$ (Supplementary Figure 2c). In order to visualize our WES data in the context of carcinogenesis, genes were grouped by major carcinogenesis pathways, namely, the RTK/Tas/PI3K, cell cycle, p53, hippo, Nrf2, or histone/chromatin modification pathway (Supplementary Figure 3). As differences in carcinogenesis mechanisms between ethnic populations have been shown, we compared our sequencing data with previously published LUAD sequencing data from predominantly western (TCGA/MSCKK) (https://gdc.cancer.gov/) and east Asian (LCCS) LUAD cohorts (23) to elucidate favorable carcinogenesis pathways among western and Asian ethnicities. As show in Supplementary Figure 3, Asian populations, both in the LCCS cohort and our study, showed relatively high incidences of upstream mutation in 

by high prevalence of EGFR mutations. Additionally, genes of the Nrf2 andp53 pathways, as well as those involved in the cell cycle, showed mutational divergences between Asian and western populations suggesting diverse carcinogenesis processes in different ethnic populations (Supplementary Figure 3). Mutation signatures across different disease stages were presented in Supplementary Figure 4a. While no significant correlations were found between stages and mutation signature, age, BRCA and ultraviolet related signatures showed decreasing trend while smoking and MMR-deficiency related signatures showed an increasing trend during tumor development (Supplementary Figure 4b). We further looked into copy number alterations (CNV) among different tumor stages. $16 p \operatorname{amp}(p=0.01), 6 q$ amp ( $=0.007), 20 q$ amp $(p=0.049), 10 q$ del $(p=0.016)$ and $12 p$ del $(p=0.023)$ alterations showed significant correlation with tumor stages which may indicate potential mechanism of chromosomal structure variation driving early tumor development (Supplementary Figure 4c). Tumor mutation burden (TMB) was significantly higher in the IAC subtype while no difference was seen between AIS and MIA subtypes (Supplementary Figure 5a). Whole genome doubling (WGD), ploidy, genomic and chromosomal instability were also evaluated across subtypes. Although not significant, increasing trend of the above features were observed through tumor development (Supplementary Figure 5a-b).

To further illustrate how genomic features may interact with immune microenvironment, we performed mIHC staining of CD8, CD56, CD68, HLA-DR and PanCK (Supplementary Figure 6a) and, correlated them with genomic characteristics. While CD8+, CD68+ and CD56+ infiltrating cells were significantly higher in tumor compared to adjacent tissues, extremely lower densities of CD8+ infiltrating cells were found in both sites, regardless of histological stage, which might be related to relatively low neoantigen burden that led to insufficient lymphocyte immunity $(29,30)$ (Supplementary Figure $6 \mathbf{b})$. Expression of PD-L1 using 22C3 assay was also performed and low PD-L1 expression (13\% for PD-L1 positive) was found in precursor LUAD, which was concordant with previous data (Supplementary Figure 6c) (18). When combining genomic features and mIHC results, we found that TMB was associated with 1q amplification, genomic instability (GII), aneuploidy and CD68+/HLA-DR+ infiltrating cells. Additionally, $8 \mathrm{q}$ amplification was associated with $\mathrm{CD} 8+$ infiltrating cells. No genomic associations were found with CD56+ infiltrating cells (Supplementary Figure 6d). These results may in part, suggest that early formation of LUAD could have undergone strong immune stress through infiltrating CD68+ macrophages, which play role in progression of preinvasive LUAD. 
1 To evaluate genomic and immune hallmarks for LUAD precursors, we derived single-cell data of malignant

2 epithelial cells defined by inferred copy number variants to avoid being confounded by data from immune cells

3 (Supplementary Figure 7). We evaluated several representative cancer hallmark gene sets involving cell

4

5 proliferation, development, DNA damage and metabolism. Proliferation, development, and metabolism gene sets showed increased expression in both adjacent lung tissue and tumor tissue, with the latter increasing linearly as pathological stage worsened. However, DNA damage response genes remained stable until onset of cancer suggesting the potential role of dysregulation in DNA damage response as a key modulator of early malignant transformation for LUAD (Figure 2a).

We also evaluated the expression of immune checkpoint ligands on tumor cells. In line with IHC data, expression of CD274 (PD-L1), one of the well-acknowledged immune checkpoints, was extremely low across all stages. However, expression of CD47, known as suppressor of innate immunity (31), was increased across all pathological stages when compared to adjacent lung tissue (Figure 2b). We applied Weighted Gene Co-Expression Network Analysis (WGCNA) (32) to identify potential genes whose expression correlated with CD47 expression in tumor cells and intersecting with TCGA LUAD data, we identified GRHL1, ZNF431 and IKZF2 as a potential transcription factor that modulates CD47 expression in LUAD precursors specifically (Supplementary Figure 8; Figure 2c). Despite widespread expression of CD47 across various cell types, expression of its receptor gene, SIRPA, was increased on macrophages (Figure 2d). Moreover, we identified a similar increasing trend of CD47 expression during different developmental stages of LUAD in mouse model (Figure 2e). Additionally, we analyzed previously published singlecell sequencing data from genetically engineered mouse model to validate our findings (Supplementary figure 9a). Integration of tumor cell single-cell sequencing data revealed a distinct evolutionary trajectory of tumor development (Supplementary figure 9b). Multiplex immunofluorescence on preinvasive LUAD from clinical samples revealed significantly higher expression of CD47 in malignant epithelial cells compared to normal alveolar epithelial cells (Figure 2f). More importantly, interaction of SIRP on tumor-infiltrating macrophages and CD47 on malignant epithelial cells was substantial at preinvasive stages (Figure 2e), making CD47 a potential candidate target to reprogram the suppressive immune microenvironment for early interception.

Intercellular Heterogeneity and Differentiation of AT2 Indicate Diverse Phenotypes for Early-stage LUAD

To exposit intercellular features during malignancy acquisition, we further look into the intercellular heterogeneity and differentiation among different stages. Since LUAD predominantly originates from alveolar type II (AT2) cells, 
1 we focused solely on AT2 cell developments. Detailed stratification by tumor stage, sample type and other

2 information of AT2 as well as functional genes of different pathways were summarized (Supplementary Figure

3 10a-b). We simultaneously applied Shannon and Simpson indices to quantify the diversity of each AT2 cell and

4

5

defined 3 subgroups based on Shannon and Simpson index values: normal, pre-malignant and malignant, which were matched to their pathological subtypes (Figure 3a) (Supplementary figure 10a). Using CytoTrace, differential potentials were calculated for each subgroup. The normal group of AT2 showed relatively lower differential potentials while malignant group showed the highest differential potentials which is concordant to a more invasive histological profile (33) (Figure 3b). By applying diversity scores to evaluate intracellular heterogeneity (ITH), we found significantly increased ITH in later histologic stages and higher diversity score in EGFR-mutant LUAD (Figure 3c). To further define marker genes that may reflect tumor biology of pre-malignant and malignant group, we screened differentially expressed genes between pre-malignant and malignant group (Figure 3d) and focused on genes specifically expressed in malignant epithelial cells. By integrating survival analysis of candidate genes in TCGA (Figure 3e), we identified MS4A15, CAPN12 and SEPIND1 as featured genes for pre-malignant group of AT2 and, KRT18 and PHLDA2 as featured genes for malignant group (Figure 3f), which might represent diverse tumor behavior in early stage LUAD. Targeting protein-coded KRT18 and PHLDA2 may potentially inhibit progression of preinvasive LUAD.

\section{Landscape of T lymphocyte and Imbalance of Th17/Treg Triggers Progression}

$\mathrm{T}$ cells were subtyped into $\mathrm{CD} 4+\mathrm{T}$ and $\mathrm{CD} 8+\mathrm{T}$ and were subsequently reclustered into several well-acknowledged subtypes (Figure 4a). To note, CD4+ T cells also contained two undefined clusters (Figure 4a). As expected, CD8+ $\mathrm{T}$ effector cells (Tef) were significantly enriched in adjacent tissue rather than tumor which is consistent with previous findings (34). We applied COSPMAP (35) to measure spatial distribution of different cell types (Supplementary Figure 11a). By calculating distance between central site and different cell types (Supplementary Figure 11b), we simulated spatial landscape of tumor cells and other major immune cell types (Supplementary Figure 11c). We hypothesize that fibroblast cells may play key roles in limiting effective T lymphocyte immunity. Indeed, multiplex fluorescence demonstrated that $\mathrm{GZMB}^{+} \mathrm{CD} 3^{+} \mathrm{CD} 8^{+} \mathrm{T}$ cells were remarkably enriched within adjacent alveolar tissues and those $\mathrm{CD} 3^{+} \mathrm{CD}^{+} \mathrm{T}$ cells were predominantly restricted by $\mathrm{SMA}^{+}$Fibroblast cells within tumor or in the boundary of tumor bed (Supplementary Figure 11d). On the contrary, CD8+ resident memory T cells (Trm) were highly enriched in tumor tissue (Supplementary Figure 12). Both Tef and Trm had high expression of GNLY, NKG7 and GZMA indicating diverse CD8+ T cell based anti-tumor mechanisms between tumor (mostly 
CD8+Trm) and adjacent tissue (mostly CD8+Tef) in early-stage LUAD (Figure 4b). Although previous studies suggested decreased infiltrating CD8+ T cells in advanced EGFR-mutant NSCLC(36), no significant difference was observed among all CD8+ T subtypes. While a small group of CD8+ exhausted T cells (Tex) was identified in precursor LUAD, these Tex also retained high expression of effective markers including NKG7, GZMA and GZMB, suggesting residule response of cytotoxic CD8+ T immunity within tumor (Figure 4b). Notably, these early-phase Tex specifically expressed CXCL13 which is known to play essential roles in recruitment of B cells. One possible explanation for upregulation of CXCL13 is that CD8+ Trm which convert to CD8+ Tex after mounting an immune response upregulate CXCL13 in order to recruit B cells to the tumor to mount an anti-tumor response $(37,38)$. Similar to CD8+ Tef cells, CD4+ Tef cells were also enriched in adjacent tissue (Supplementary Figure 13). Of note, both T helper 17 (Th17) and CD4+ T regulator cells (Treg) were enriched within tumor tissue (Supplementary

Figure 13). By performing pseudo-time analysis to visualize evolution of CD4+ T cells and integrating distribution of CD4+ T cells from different histologic stages, we found a trend towards higher proportion of Treg cells during disease progression while inflammatory Th17 cells remained similar (Figure 4c). Therefore, we further assessed Th17/Treg ratio in tumor and tumor adjacent tissue from patients with different histologic stages. The results demonstrated comparable Th17/Treg ratio in adjacent tissue across different histologic stages, but significantly lower Th17/Treg ratio $(\mathrm{p}=0.031)$ in later histologic stages (Figure 4d). These results suggest a change of balance of Th17/Treg as a major immunoregulatory event during progression of preinvasive LUAD.

\section{Limited Expansion of Intra-tumoral T Lymphocytes across stages}

The T cell receptor (TCR) was the key component that reflects interaction between T cells and cancer cells (39). So far, no studies evaluated the degree of interaction between T cells and cancer cells among such early initiative disease. Interestingly, by integrating scRNA-seq and scTCR-seq, we found that most of the T cells both in tumor and adjacent tissue were relatively static and presented as single clones (Figure 4e) (40). Regarding different subtypes of CD4+ and CD8+ T cells, relatively high expansion of TCR in CD4+/CD8+ Tef within adjacent tissue was identified while significantly high expansion of CD4+ Treg and CD4+ Trm were observed within tumor (Figure 4e). Based on different distribution of TCR and clone size, we further established the parallel clonal size according to different clusters. Although clonal size of CD8+ Tef exhibited significant correlation between tumor and adjacent clone sizes, tumor-specific TCR expansion was extremely low as well as CD4+Tef, suggesting limited adaptive anti-tumor immune response at LUAD precursor stages. For Treg, no significant correlation of clonal size was found between tumor and adjacent tissue IT (Supplementary figure 14). Furthermore, we evaluate TCR diversity among different 
cell types. Only CD4+Treg and Trm of tumor showed significantly higher TCR diversity compared to adjacent tissue. Taken together, these results revealed limited response of adaptive immunity during early initiation of LUAD and highlighted immune suppression of Treg during invasiveness acquisition in LUAD precursor stages.

\section{LAMP3+DC as Double-edged Immune Modulator During Early Progression}

Given that dendritic cells (DCs) play essential roles in innate immunity and antigen presentation, we next sought to explore the dynamic changes of DCs during early neoplastic progression of LUAD. Multiple clusters of DCs were defined based on marker genes and their distribution among adjacent and tumor tissue, as well as histological stages, was summarized in Supplementary Figure 15a. Notably, LAMP3+ DC and CD163+ DC clusters contained unique expression profiles (Supplementary Figure 15b). While CD163+ DC presented relatively high expression of a wide range pro-inflammatory chemokines such as CCL3, CXCL8 and CCL20, LAMP3+ DC showed high expression of CCL19 and CCL17, cytokines that are known to promote recruitment for T lymphocyte infiltration. Additionally, LAMP3+ DC specifically expressed CD274 (PD-L1) and CD200 which are known to suppress immune responses (Supplementary Figure 15b). However, LAMP3+ DC also specifically expressed IL15 and IL32which induce strong anti-tumor immune responses by stimulating proliferation of $\mathrm{T}$ cells, B cells and NK cells (41) (Supplementary Figure 15b). To clarify the relationship between different subtypes of DCs, we used PAGA to construct an evolutionary model (Supplementary Figure 15c). While pDCs were relatively separated, both CD1C+ DC and CLEC9A+ DCs appeared to transform directly to LAMP3+ DC, a finding which is consistent with previous studies (41). Using functional gene sets from well-defined panels, we defined immune functions for each cluster. LAMP3 + DC had highest expression of genes associated with immune regulation, migration and Th2 response which supported the findings of their expression profile discussed above (Supplementary Figure 15d). Although not statistically significant, LAMP3+ DC infiltration was higher in MIA and IAC subtypes when compared to AIS subtype, which could suggest a role of LAMP3+ DCs in immune suppression during progression of LUAD (Supplementary Figure 15e, Figure 5b).

\section{Interplay of Cancer Cells and Immune Cells Upon Different Stages}

To comprehensively visualize the interaction between malignant cells and immune cells, we applied Cellphone DB to explicate interplay communication among tumor cells and immune cells. We found increased interaction of CXCL17-GPR35 axis between tumor cells and CD4+/CD8+ T cells, which have been shown to correlate with tumor progression and metastasis (42) (Figure 5a). Additionally, a well acknowledged immune checkpoint, HAVCR2 
1 (TIM3), (43) specifically expressed on myeloid cells was significantly increased during neoplastic evolution through

2 binding to its receptor LGALS9 on tumor cells. Another important interaction was between CSF2, which plays

3 pivotal roles in recruitment of macrophages (44), and VEGFB which modulates angiogenesis during invasiveness

4 acquisition (Figure 5a).

5 Then, we established an interaction network of immune cells across histological stages to further illustrate immune

6 features of different stages (Figure 5b). The network model was divided into early, intermediate and late stages regarding percentage of cellular components among different stages. During early stage, CD8+ Tef was activated though they were mostly rejected without effectively infiltrating into the tumors based diverse distribution of CD8+Tef between tumor and adjacent tissues. Meanwhile, increased levels of conventional immune cells, including CD8+ MAIT, NK, and alveolar macrophages, were identified within early-stage tumors. When LUAD evolved into intermediate stage, DCs started to interfere extensively and shape immune environment. During late stage, there was a significant increase in infiltrating B cells and other lymphocytes, suggesting formation of tertiary lymphoid structure (TLS) during invasive acquisition. Distribution of B cells also showed increased tumor infiltrating B cells upon different histologic stages (Supplementary Figure 15). Besides, as illustrated above, Treg was also highly interacted and enriched in invasive LUAD, while Th17 remained similar across different histologic stages indicating the dynamic changes in the balance of Th17/Treg during neoplastic progression (Figure 5b). We next evaluated the frequencies of ligand-receptor interaction among immune cells. By clustering cells into different modules based on variation trend, we identified 3 modules which showed significant correlation with stages (Figure 5c). We selected representative interaction with over $40 \%$ in either up-regulatory module or down-regulatory module to analyze further. For upregulated ligand-receptor interactions enhanced along with stages, increased recruitment of Treg as well as B cells was observed through interaction with myeloid cells. For down-regulated receptor-ligand interactions, decreased recruitment for macrophages and Tef were found (Figure 5d), indicating an immune-modulated blueprint during early carcinogenesis of LUAD.

To comprehensively visualize the interplay between cancer cells and immune cells, we established an interactive network diagrammatic sketch and described featured immune involvement upon stages along with intrinsic molecular events of cancer cells among different tumor stages (Figure 6).

\section{Discussion}

Herein, we performed a deep multi-omics analysis on predominantly preinvasive as well as early invasive LUAD, and identified unique genomic and immune features associated with the early neoplastic progression of LUAD. 
1 Based on our previous finding (28), we further integrate genomic and immune microenvironment features, and results suggested that infiltrating macrophages might act as key modulator for tumor cells by increasing genomic complexity, which is concordant to the findings of elevated CD47 on malignant epithelial cells in later stages. Cancer hallmarks represent various featured aspects that promote tumor survival and proliferation (45). Similar in preinvasive lung squamous cell carcinoma (46), we also observed increased level of multiple functional pathways including cell proliferation and metabolism. Additionally, we identified remarkably increased expression of DNA damage repair relevant pathways which may indicate critical molecular events of malignant transformation, particularly in non-smoking Asian population. Multiple studies showed relatively low intra-tumoral heterogeneity of early-stage lung adenocarcinoma through bulk sequencing $(28,47,48)$. Taking advantage of single cell technique, we further explored intercellular heterogeneity and tumor plasticity during initiation and early progression of LUAD. We found extremely high intercellular heterogeneity even at the precancer stage. Furthermore, EGFR mutant-LUAD precursors showed significantly higher tumor diversity consistent with previous research (7). By analyzing differentially expressed genes and integrating external datasets, we also found two protein-coded genes KRT18 and PHLDA2 as potential targets for early interception to prevent malignant transformation and progression. In contrast to advanced NSCLC whereas PD-L1 positive rate could be more than $60 \%$ with around $30 \%$ showing high PD-L1 expression (defined as tumor proportion score, TPS $>50 \%$ ) (49-52), previous studies suggested weak PD-L1 expression in early-stage NSCLC tumor, especially for preinvasive LUAD (28). In this study, only $13.0 \%$ (7/54) of LUAD precursors were positive for PD-L1, none of which with high PD-L1 expression. Recent study showed limited efficacy of PD-1 blockade for sub-solid nodules (53), which may represent preinvasive or early malignant LUAD suggesting other immune escape mechanisms may exist at preinvasive stages (54). By integrating large-scale high-quality single-cell sequencing data of both adjacent and tumor tissues, normal lung tissues from healthy donors and external datasets, we discovered that elevated CD47 expression may play essential roles in immune evasion at preinvasive stage. Meanwhile, SIRPA expression was specifically expressed in macrophages which could interact with CD47 and prevent innate anti-tumor immunity. Further results of multiplex fluorescence demonstrated interaction of CD47 and SIRP between infiltrating macrophages and malignant epithelial cells. Besides, we also found several potential targets such as ZNF431, GRHL1 and IKZF2 that may be involved in modulating CD47 expression at preinvasive stages of LUAD. Multiple preclinical studies and phase I trials evaluated anti-CD47 in advanced cancers (55-57). Our findings suggest that these CD47-targeting agents could be considered as early interception for preinvasive LUAD by enhancing innate anti-tumor immunity to prevent acquisition of invasiveness. 
barely infiltrated within tumor in our cohort. Given that increased infiltrating CD4 T cells, we further looked into evolution trajectory of CD4 lymphocytes in different histologic stages and revealed imbalance of Th17/Treg that may be essential during disease progression. Previous study also reported that compared to solid LUAD, SSNassociated LUAD manifested higher Treg(58). In our study, single-cell TCR data showed relatively inactive status of T lymphocytes, suggesting that lymphocyte immunity stimulation rather than relieving lymphocyte suppression by PD-1/PD-L1 blockade may be more effective therapeutic approach for these LUAD precursors.

As another key component of innate immunity, we found a unique group of LAMP ${ }^{+}$DC that has been previously described in other cancer types $(25,59)$ may have played important roles during early neoplastic evolution of LUAD. These $\mathrm{LAMP}^{+}$DC originated from $\mathrm{CD}^{+} \mathrm{C}^{+}$and $\mathrm{CLEC} \mathrm{A}^{+}$DCs. Unlike other DC subtypes, the LAMP3 ${ }^{+}$DC expressed high immunoregulatory related genes without conventional antigen presenting function despite high expression of CCL19 and CCL17. Through ligand-receptor interaction between tumor cells and immune cells, we identified strong interaction of CXCL17-GPR35 axis in various histologic stages. CXCL17-GPR35 axis has been reported to promote tumor proliferation and metastasis in breast cancer (60).

Up to date, surgery remains as the standard treatment for early-stage NSCLC. However, as local treatment, it may not be adequate to prevent local or distant metastasis $(61,62)$. Moreover, increasing patients were found to have multi-focal lung cancer making surgical resection challenging. Despite encouraging results of neoadjuvant PD-1/PDL1 blockade presented in recent years (63-65), such treatment modality has shown suboptimal efficacy for preinvasive or early invasive LUAD. Our study suggested that relieving inactive innate immunity to enhance antitumor immunity for these LUAD precursors may be more effective. We have also identified KRT18 and PHLDA2 as two potentially novel targets for interception at precursor stages. These targets warrant further investigation including using preclinical models to clarify their clinical value. Moreover, our study provided a comprehensive genomic and immune microenvironment landscape of LUAD precursors, and established an interactive network of LUAD precursors of consecutive developmental stages. To maximize the benefit of these deep-profiling data to the lung cancer research community, we generated an interactive portal for our datasets, which could be applied as a resource for further exploration of diagnostic and therapeutic targets as well as infiltrating immune cells in preinvasive LUAD.

\section{Experimental model and subject details}

\section{Human Tumor Samples}

Written informed consent of tumor acquisition for research was obtained before surgery and approved by internal 
China, IRB approved protocol number GDREC2016175H).54 surgically resected sub-solid nodules pathologically diagnosed as lung adenocarcinoma were collected. Nodules were defined as sub-solid lesions if at least one of the following conditions was met: 1) no solid components were found under the mediastinal window or 2) nodules with partial solid lesions under the mediastinal window contained ground glass components. Details of sample acquisition and processing process was presented in Supplementary figure 1a-b. Briefly, portions of each resected nodule were immediately used for downstream analysis (freshly resected) and preserved in frozen, and formalin-fixed and paraffin-embedded (FFPE) tissue blocks. Freshly resected tissues used for single cell sequencing were prepared as single cell suspensions and sequenced immediately. Frozen blocks were stored under $-80^{\circ} \mathrm{C}$ and parrffin- embedded blocks were stored under room temperature. Both frozen and paraffin-embedded samples were sent for whole exome sequencing (WES) and multiplex immunohistochemistry (mIHC) within a few months of preservation. Quality control analyses for single-cell and bulk sequencing, including cell viability, median genes per cell and mean sequencing depth, were performed (Supplementary figure 1c-f).

\section{Histology Evaluation}

FFPE slide of each tumor samples were stained with H\&E. Hematoxylin-eosin (HE) and thyroid transcription factor1 (TTF-1) staining was performed on all samples to confirm pathology. Histological evaluation was performed by two specialized pathologists. Adenocarcinoma in situ (AIS) was defined as 100\% lepidic patterns without invasive components. Minimally invasive adenocarcinoma (MIA) was defined as lepidic predominant patterns with invasive component less than $0.5 \mathrm{~cm}$. Invasive adenocarcinoma (IAC) was defined as mixed pathological patterns with tumor involvement through basement membrane. We also evaluated thyroid transcription factor 1(TTF-1) among all samples considering its high specialty in lung adenocarcinoma and prognostic value.

\section{Multiplex Immunohistochemistry Staining and Analysis}

To identify the cell subsets in the TME, 5 slides from 5-um-thick non-stained formalin-fixed and paraffin-embedded samples underwent multiplex immunofluorescence staining using the PANO 7-plex IHC kit (cat 0004100100, Panovue, Beijing, China) and primary antibodies of panel 1 (CD68, CD163, CD47, SIRP and PANCK) and panel 2 (CD3, CD8A, GZB, CD56, SMA and PANCK). After labeling with primary antibodies, samples were treated with a horseradish peroxidase-conjugated secondary antibody followed by tyramide signal amplification (TSA). Slides were microwave heat-treated after each TSA operation. To visualize nuclei, slides were stained with 4'-6'-diamidino- 
1 2-phenylindole (DAPI, SIGMA-ALDRICH) after antibody labeling. To obtain multispectral images, the stained

2 slides were scanned using the Mantra System (PerkinElmer, Waltham, Massachusetts, US), which captures the

3 fluorescent spectra at 20-nm wavelength intervals from 420 to $720 \mathrm{~nm}$ with identical exposure time; the scans were

4 combined to build a single stack image.

5 Images of unstained and single-stained sections were used to extract the spectrum of autofluorescence of tissues and each fluorescein, respectively. The extracted images were further used to establish a spectral library required for multispectral unmixing by inForm image analysis software 2.2.1. (PerkinElmer, Waltham, Massachusetts, US). Using this spectral library, we generated reconstructed images of sections with the autofluorescence removed.

\section{Single-cell Dissociation}

Single-cell RNA-seq experiments were performed by experimental personnel in the laboratory of NovelBio Co.,Ltd. The tissues were surgically removed and kept in MACS Tissue Storage Solution (Miltenyi Biotec) until processing. The tissue samples were processed as described below. Briefly, samples were washed with phosphate-buffered saline (PBS), minced into small pieces (approximately 1mm3) on ice, and enzymatically digested with $\mathrm{xx} \mathrm{U} / \mathrm{mL}$ collagenase I (Worthington) and xx U/mL DNase I (Worthington) for $45 \mathrm{~min}$ at $37^{\circ} \mathrm{C}$, with agitation. After digestion, samples were sieved through a $70 \mu \mathrm{m}$ cell strainer, and centrifuged at $300 \mathrm{~g}$ for $5 \mathrm{~min}$. After the supernatant was removed, the pelleted cells were suspended in red blood cell lysis buffer (Miltenyi Biotec)and incubated at room temperature until all red blood cells were lysed (approximately 12 minutes). After washing with PBS containing 0.04\% BSA, the cells were re-suspended in PBS containing 0.04\% BSA and re-filtered through a $35 \mu \mathrm{m}$ cell strainer. Dissociated single cells were then stained with AO/PI for viability assessment using Countstar Fluorescence Cell Analyzer. The single-cell suspension was further enriched with a MACS dead cell removal kit (Miltenyi Biotec).

\section{Single-cell RNA Sequencing}

The scRNA-Seq libraries were generated using the 10X Genomics Chromium Controller Instrument and Chromium Single Cell 3'V3 Reagent Kits (10X Genomics, Pleasanton, CA). Briefly, cells were concentrated to 1000 cells/uL and approximately 8,000 cells were loaded into each channel to generate single-cell Gel Bead-In-Emulsions (GEMs), which results into expected mRNA barcoding of 5,000 single-cells for each sample. After the RT step, GEMs were broken and barcoded-cDNA was purified and amplified. The amplified barcoded cDNA was fragmented, A-tailed, ligated with adaptors and index PCR amplified. The final libraries were quantified using the Qubit High Sensitivity DNA assay (Thermo Fisher Scientific) and the size distribution of the libraries were determined using a High 
Sensitivity DNA chip on a Bioanalyzer 2200 (Agilent). All libraries were sequenced by illumina sequencer (Illumina, San Diego, CA) on a 150 bp paired-end run.

\section{Single-cell TCR Sequencing}

The scRNA-Seq libraries and V(D)J libraries were generated using the 10X Genomics Chromium Controller Instrument and Chromium Single Cell 5' library \& gel bead kit, along with the V(D)J enrichment kit (10X Genomics, Pleasanton, CA). Briefly, cells were concentrated to 1000 cells/uL and approximately 8,000 cells were loaded into each channel to generate single-cell Gel Bead-In-Emulsions (GEMs), which results into expected mRNA barcoding of 5,000 single-cells for each sample. After the RT step, GEMs were broken and barcoded-cDNA was purified and amplified. The amplified barcoded cDNA was used to construct 5' gene expression libraries and TCR enriched libraries. For 5' library construction, the amplified barcoded cDNA was fragmented, A-tailed, ligated with adaptors and index PCR amplified. For the V(D)J library, human T cell V(D)J sequences were enriched from the amplified cDNA followed by fragmentation, A-tailing, adaptor ligation and index PCR amplification. The final libraries were quantified using the Qubit High Sensitivity DNA assay (Thermo Fisher Scientific) and the size distribution of the libraries were determined using a High Sensitivity DNA chip on a Bioanalyzer 2200 (Agilent). All libraries were sequenced by illumina sequencer (Illumina, San Diego, CA) on a 150 bp paired-end run.

\section{Single-cell RNA Statistical Analysis}

scRNA-seq data analysis was performed by NovelBio Co.,Ltd. with NovelBrain Cloud Analysis Platform (www.novelbrain.com). We applied fastp (66) with default parameter filtering the adaptor sequence and removed the low quality reads to achieve the clean data. Then the feature-barcode matrices were obtained by aligning reads to the human genome (GRCh38 Ensemble: version 91) using CellRanger v3.1.0. We applied the down sample analysis among samples sequenced according to the mapped barcoded reads per cell of each sample and finally achieved the aggregated matrix. Cells which contained over 200 expressed genes and had mitochondria UMI rates below $20 \%$ passed the cell quality filtering and mitochondria genes were removed in the expression table.

Seurat package (version: 2.3.4, https://satijalab.org/seurat/) was used for cell normalization and regression based on the expression table according to the UMI counts of each sample and percent of mitochondria rate to obtain the scaled data. PCA was constructed based on the scaled data with top 2000 high variable genes and top 10 principals were used for tSNE construction and UMAP construction. CCA analysis in Seurat package was applied for combining 3' scRNA-Seq data and 5' scRNA-Seq data to correct batch effect. Utilizing graph-based cluster method, 
1 we acquired the unsupervised cell cluster result based the PCA top 10 principal and we calculated the marker genes

2 by FindAllMarkers function with wilcox rank sum test algorithm under following criteria: $1 . \operatorname{lnFC}>0.25 ; 2$.

3 pvalue $<0.05 ; 3$. min.pct $>0.1$. In order to identify the cell type detailed, the clusters of same cell type were selected

4 for re-tSNE analysis, graph-based clustering and marker analysis.

5

\section{Diversity score}

7 We applied predefined index called diversity score to measure the degree of intratumoral heterogeneity(67). As previously described, diversity score of the tumor was defined as the average distance of all malignant cells within the tumor to the centroid, which could be calculated as

$$
\text { Diversity score }=\frac{1}{m} \sum_{i=1}^{m} \sqrt{\sum_{j=1}^{n}\left(X_{i j}-\mu_{j}\right)^{2}}
$$

\section{Pseudo-Time Analysis}

We applied the Single-Cell Trajectories analysis utilizing Monocle2 (http://cole-trapnell-lab.github.io/monoclerelease) using DDR-Tree and default parameter. Before Monocle analysis, we select marker genes of the Seurat clustering result and raw expression counts of the cell passed filtering. Based on the pseudo-time analysis, branch expression analysis modeling (BEAM Analysis) was applied for branch fate determined gene analysis.

More Single-Cell Trajectories analysis was applied by utilizing PAGA in scanpy package (https://bioconductor.org/packages/release/bioc/html/slingshot.html version 1.4.0). Before analysis, we select marker genes of the Seurat clustering result and raw expression counts of the cell passed filtering.

\section{Cell Communication Analysis}

To enable a systematic analysis of cell-cell communication molecules, we applied cell communication analysis based on the CellPhoneDB (68), a public repository of ligands, receptors and their interactions. Membrane, secreted and peripheral proteins of the cluster at different time points were annotated. Significant mean of different cell subsets proportion and Cell Communication significance $(\mathrm{p}$-value $<0.05)$ were calculated based on the interaction and the normalized cell matrix achieved by Seurat Normalization. More cell communication was applied based on the cell expression data by Cellchat (69) (https://github.com/sqjin/CellChat, version: 0.0.2) 


\section{CNV Estimation}

2 Cells defined as endothelia, fibroblast and macrophage were used as reference to identify somatic copy number 3 variations with the R package infercnv (v0.8.2). We scored each cell for the extent of CNV signal, defined as the mean of squares of CNV values across the genome. Putative malignant cells were then defined as those with CNV signal above 0.05 and $\mathrm{CNV}$ correlation above 0.5 .

\section{Go Analysis}

Gene ontology (GO) analysis (70) was performed to facilitate elucidating the biological implications of marker genes and differentially expressed genes. We downloaded the GO annotations from NCBI (http://www.ncbi.nlm.nih.gov/), UniProt (http://www.uniprot.org/) and the Gene Ontology (http://www.geneontology.org/). Fisher's exact test was applied to identify the significant GO categories and FDR was used to correct the p-values.

\section{Pseudo-cell analysis and WGCNA analysis}

In order to achieve expression matrix for WGCNA analysis (Weighted-Gene Co-Expression Network Analysis), pseudo-cell analysis was applied based on the single cell data by schex (v:1.0.55). We utilized pseudo-cell expression matrix to construct the WGCNA signed network with the beta score as 8 for module clustering.

\section{Statistical analysis}

All statistical analysis was performed in SPSS version 25.0 software (SPSS, Inc., Chicago, IL, USA). t-tests were used for two group comparison and ANOVA or Kruskal-Wallis tests were used for more than two group comparisons. Fisher's exact test was used to compare two categorical variables. Kaplan Meier analysis was used to compare survival between groups and log-rank test $\mathrm{p}$ value was calculated.

\section{Acknowledgements}

We thank Geneseeq technology for providing high-throughput whole exome sequencing and $\mathrm{T}$ cell receptor repertoire sequencing. We thank NovelBio for providing single cell RNA sequencing and bioinformatic analysis support. This study was supported by National Natural Science Foundation (grant 81872510 to Dr. Zhong), Highlevel Hospital Construction Project (grant DFJH201801 to Dr. Zhong).

\section{Author contributions}


Conceptualization, C.Z., W.Z., and Y.W.; Resources, C.Z., Y.S., Z.X., and L.Y.; Methodology, C.Z., Y.S., Z.Q., H.H., Z.Q., and L.Y.; Bioinformatic Analysis, C.Z., S.Y.; Writing-Original Draft, C.Z.; Writing-Review \& Editing, C.Z., S.Y., W.Z., Z.Q., J. Y., J.Z., and Y.W.

\section{Data Availability}

The datasets generated from single cell RNA sequencing in this study are available at GEO through their respective accession number GSE164789. Detail measurement data of mIHC and MAF files of WES were all summarized in Supplementary Data. All other relevant data is available from corresponding author (Wen-zhao Zhong, syzhongwenzhao@scut.edu.cn) of this study upon reasonable request.

\section{Reference}

1. Ribas A, Wolchok JD. Cancer immunotherapy using checkpoint blockade. Science (New York, NY). 2018;359(6382):1350-5.

2. Hirsch FR, Scagliotti GV, Mulshine JL, Kwon R, Curran WJ, Jr., Wu YL, et al. Lung cancer: current therapies and new targeted treatments. Lancet (London, England). 2017;389(10066):299-311.

3. Siegel RL, Miller KD, Jemal A. Cancer statistics, 2020. CA: a cancer journal for clinicians. 2020;70(1):7-30.

4. Howlader N, Forjaz G, Mooradian MJ, Meza R, Kong CY, Cronin KA, et al. The Effect of Advances in Lung-Cancer Treatment on Population Mortality. The New England journal of medicine. 2020;383(7):640-9.

5. Aberle DR, Adams AM, Berg CD, Black WC, Clapp JD, Fagerstrom RM, et al. Reduced lungcancer mortality with low-dose computed tomographic screening. The New England journal of medicine. 2011;365(5):395-409.

6. Jamal-Hanjani M, Wilson GA, McGranahan N, Birkbak NJ, Watkins TBK, Veeriah S, et al. Tracking the Evolution of Non-Small-Cell Lung Cancer. The New England journal of medicine. 2017;376(22):2109-21.

7. Nahar R, Zhai W, Zhang T, Takano A, Khng AJ, Lee YY, et al. Elucidating the genomic architecture of Asian EGFR-mutant lung adenocarcinoma through multi-region exome sequencing. Nature communications. 2018;9(1):216.

8. Zhang XC, Wang J, Shao GG, Wang Q, Qu X, Wang B, et al. Comprehensive genomic and immunological characterization of Chinese non-small cell lung cancer patients. Nature communications. 2019;10(1):1772.

9. Swanton C. Intratumor heterogeneity: evolution through space and time. Cancer research. 2012;72(19):4875-82.

10. Abbosh C, Birkbak NJ, Wilson GA, Jamal-Hanjani M, Constantin T, Salari R, et al. Phylogenetic ctDNA analysis depicts early-stage lung cancer evolution. Nature. 2017;545(7655):446-51.

11. Sequist LV, Waltman BA, Dias-Santagata D, Digumarthy S, Turke AB, Fidias P, et al. Genotypic and histological evolution of lung cancers acquiring resistance to EGFR inhibitors. Science translational medicine. 2011;3(75):75ra26. 
12. Travis WD, Brambilla E, Noguchi M, Nicholson AG, Geisinger KR, Yatabe Y, et al. International association for the study of lung cancer/american thoracic society/european respiratory society international multidisciplinary classification of lung adenocarcinoma. Journal of thoracic oncology: official publication of the International Association for the Study of Lung Cancer. 2011;6(2):24485.

13. Gao JW, Rizzo S, Ma LH, Qiu XY, Warth A, Seki N, et al. Pulmonary ground-glass opacity: computed tomography features, histopathology and molecular pathology. Translational lung cancer research. 2017;6(1):68-75.

14. Hattori A, Suzuki K, Takamochi K, Wakabayashi M, Aokage K, Saji H, et al. Prognostic impact of a ground-glass opacity component in clinical stage IA non-small cell lung cancer. The Journal of thoracic and cardiovascular surgery. 2021;161(4):1469-80.

15. Shewale JB, Nelson DB, Rice DC, Sepesi B, Hofstetter WL, Mehran RJ, et al. Natural History of Ground-Glass Lesions Among Patients With Previous Lung Cancer. The Annals of thoracic surgery. 2018;105(6):1671-7.

16. Hiramatsu M, Inagaki T, Inagaki T, Matsui Y, Satoh Y, Okumura S, et al. Pulmonary groundglass opacity (GGO) lesions-large size and a history of lung cancer are risk factors for growth. Journal of thoracic oncology : official publication of the International Association for the Study of Lung Cancer. 2008;3(11):1245-50.

17. Chang B, Hwang JH, Choi YH, Chung MP, Kim H, Kwon OJ, et al. Natural history of pure ground-glass opacity lung nodules detected by low-dose CT scan. Chest. 2013;143(1):172-8.

18. Zhang C, Zhang J, Xu FP, Wang YG, Xie Z, Su J, et al. Genomic Landscape and Immune Microenvironment Features of Preinvasive and Early Invasive Lung Adenocarcinoma. Journal of thoracic oncology : official publication of the International Association for the Study of Lung Cancer. 2019;14(11):1912-23.

19. Dejima H, Hu X, Chen R, Zhang J, Fujimoto J, Parra ER, et al. Immune evolution from preneoplasia to invasive lung adenocarcinomas and underlying molecular features. Nature communications. 2021;12(1):2722.

20. Hu X, Estecio MR, Chen R, Reuben A, Wang L, Fujimoto J, et al. Evolution of DNA methylome from precancerous lesions to invasive lung adenocarcinomas. Nature communications. 2021;12(1):687.

21. Hu X, Fujimoto J, Ying L, Fukuoka J, Ashizawa K, Sun W, et al. Multi-region exome sequencing reveals genomic evolution from preneoplasia to lung adenocarcinoma. Nature communications. 2019;10(1):2978.

22. Chen H, Carrot-Zhang J, Zhao Y, Hu H, Freeman SS, Yu S, et al. Genomic and immune profiling of pre-invasive lung adenocarcinoma. Nature communications. 2019;10(1):5472.

23. Zheng $\mathrm{C}$, Zheng L, Yoo JK, Guo H, Zhang Y, Guo X, et al. Landscape of Infiltrating T Cells in Liver Cancer Revealed by Single-Cell Sequencing. Cell. 2017;169(7):1342-56.e16.

24. Guo X, Zhang Y, Zheng L, Zheng C, Song J, Zhang Q, et al. Global characterization of T cells in non-small-cell lung cancer by single-cell sequencing. Nature medicine. 2018;24(7):978-85.

25. Zhang L, Li Z, Skrzypczynska KM, Fang Q, Zhang W, O'Brien SA, et al. Single-Cell Analyses Inform Mechanisms of Myeloid-Targeted Therapies in Colon Cancer. Cell. 2020;181(2):442-59.e29. 26. Xing X, Yang F, Huang Q, Guo H, Li J, Qiu M, et al. Decoding the multicellular ecosystem of lung adenocarcinoma manifested as pulmonary subsolid nodules by single-cell RNA sequencing. Science advances. 2021;7(5). 
27. Shen R, Seshan VE. FACETS: allele-specific copy number and clonal heterogeneity analysis tool for high-throughput DNA sequencing. Nucleic acids research. 2016;44(16):e131.

28. Zhang C, Zhang J, Xu FP, Wang YG, Xie Z, Su J, et al. Genomic Landscape and Immune Microenvironment Features of Preinvasive and Early-Invasive Lung Adenocarcinoma. Journal of thoracic oncology : official publication of the International Association for the Study of Lung Cancer. 2019.

29. Wang D, Pham NA, Freeman TM, Raghavan V, Navab R, Chang J, et al. Somatic Alteration Burden Involving Non-Cancer Genes Predicts Prognosis in Early-Stage Non-Small Cell Lung Cancer. Cancers. 2019;11(7).

30. McGranahan N, Furness AJ, Rosenthal R, Ramskov S, Lyngaa R, Saini SK, et al. Clonal neoantigens elicit $T$ cell immunoreactivity and sensitivity to immune checkpoint blockade. Science (New York, NY). 2016;351(6280):1463-9.

31. Feng M, Jiang W, Kim BYS, Zhang CC, Fu YX, Weissman IL. Phagocytosis checkpoints as new targets for cancer immunotherapy. Nature reviews Cancer. 2019;19(10):568-86.

32. Langfelder P, Horvath S. WGCNA: an R package for weighted correlation network analysis. BMC bioinformatics. 2008;9:559.

33. Gulati GS, Sikandar SS, Wesche DJ, Manjunath A, Bharadwaj A, Berger MJ, et al. Single-cell transcriptional diversity is a hallmark of developmental potential. Science (New York, NY). 2020;367(6476):405-11.

34. Reuben A, Zhang J, Chiou SH, Gittelman RM, Li J, Lee WC, et al. Comprehensive T cell repertoire characterization of non-small cell lung cancer. Nature communications. 2020;11(1):603. 35. Ren X, Zhong G, Zhang Q, Zhang L, Sun Y, Zhang Z. Reconstruction of cell spatial organization from single-cell RNA sequencing data based on ligand-receptor mediated self-assembly. Cell research. 2020;30(9):763-78.

36. Dong ZY, Zhang JT, Liu SY, Su J, Zhang C, Xie Z, et al. EGFR mutation correlates with uninflamed phenotype and weak immunogenicity, causing impaired response to PD-1 blockade in non-small cell lung cancer. Oncoimmunology. 2017;6(11):e1356145.

37. Ganesan AP, Clarke J, Wood O, Garrido-Martin EM, Chee SJ, Mellows T, et al. Tissue-resident memory features are linked to the magnitude of cytotoxic $\mathrm{T}$ cell responses in human lung cancer. Nature immunology. 2017;18(8):940-50.

38. Mueller SN, Mackay LK. Tissue-resident memory T cells: local specialists in immune defence. Nature reviews Immunology. 2016;16(2):79-89.

39. Schrama D, Ritter C, Becker JC. T cell receptor repertoire usage in cancer as a surrogate marker for immune responses. Seminars in immunopathology. 2017;39(3):255-68.

40. Wu TD, Madireddi S, de Almeida PE, Banchereau R, Chen YJ, Chitre AS, et al. Peripheral T cell expansion predicts tumour infiltration and clinical response. Nature. 2020;579(7798):274-8.

41. Zhang Q, He Y, Luo N, Patel SJ, Han Y, Gao R, et al. Landscape and Dynamics of Single Immune Cells in Hepatocellular Carcinoma. Cell. 2019;179(4):829-45.e20.

42. Hsu YL, Yen MC, Chang WA, Tsai PH, Pan YC, Liao SH, et al. CXCL17-derived CD11b(+)Gr$1(+)$ myeloid-derived suppressor cells contribute to lung metastasis of breast cancer through platelet-derived growth factor-BB. Breast cancer research : BCR. 2019;21(1):23.

43. Acharya N, Sabatos-Peyton C, Anderson AC. Tim-3 finds its place in the cancer immunotherapy landscape. Journal for immunotherapy of cancer. 2020;8(1).

44. Zhang M, Liu F, Zhou P, Wang Q, Xu C, Li Y, et al. The MTOR signaling pathway regulates 
macrophage differentiation from mouse myeloid progenitors by inhibiting autophagy. Autophagy. 2019;15(7):1150-62.

45. Hanahan D, Weinberg RA. Hallmarks of cancer: the next generation. Cell. 2011;144(5):64674.

46. Mascaux C, Angelova M, Vasaturo A, Beane J, Hijazi K, Anthoine G, et al. Immune evasion before tumour invasion in early lung squamous carcinogenesis. Nature. 2019;571(7766):570-5.

47. Zhang J, Fujimoto J, Zhang J, Wedge DC, Song X, Zhang J, et al. Intratumor heterogeneity in localized lung adenocarcinomas delineated by multiregion sequencing. Science (New York, NY). 2014;346(6206):256-9.

48. Wang S, Du M, Zhang J, Xu W, Yuan Q, Li M, et al. Tumor evolutionary trajectories during the acquisition of invasiveness in early stage lung adenocarcinoma. Nature communications. 2020;11(1):6083.

49. Borghaei H, Paz-Ares L, Horn L, Spigel DR, Steins M, Ready NE, et al. Nivolumab versus Docetaxel in Advanced Nonsquamous Non-Small-Cell Lung Cancer. The New England journal of medicine. 2015;373(17):1627-39.

50. Brahmer J, Reckamp KL, Baas P, Crino L, Eberhardt WE, Poddubskaya E, et al. Nivolumab versus Docetaxel in Advanced Squamous-Cell Non-Small-Cell Lung Cancer. The New England journal of medicine. 2015;373(2):123-35.

51. Garon EB, Rizvi NA, Hui R, Leighl N, Balmanoukian AS, Eder JP, et al. Pembrolizumab for the treatment of non-small-cell lung cancer. The New England journal of medicine. 2015;372(21):2018-28.

52. Reck M, Rodriguez-Abreu D, Robinson AG, Hui R, Csoszi T, Fulop A, et al. Pembrolizumab versus Chemotherapy for PD-L1-Positive Non-Small-Cell Lung Cancer. The New England journal of medicine. 2016;375(19):1823-33.

53. Zhang C, Yin K, Liu SY, Yan LX, Su J, Wu YL, et al. Multiomics analysis reveals a distinct response mechanism in multiple primary lung adenocarcinoma after neoadjuvant immunotherapy. Journal for immunotherapy of cancer. 2021;9(4).

54. Wu F, Li W, Zhao W, Zhou F, Xie H, Shi J, et al. Synchronous ground-glass nodules showed limited response to anti-PD-1/PD-L1 therapy in patients with advanced lung adenocarcinoma. 2020;10(3):e149.

55. Sikic BI, Lakhani N, Patnaik A, Shah SA, Chandana SR, Rasco D, et al. First-in-Human, Firstin-Class Phase I Trial of the Anti-CD47 Antibody Hu5F9-G4 in Patients With Advanced Cancers. Journal of clinical oncology : official journal of the American Society of Clinical Oncology. 2019;37(12):946-53.

56. Gholamin S, Mitra SS, Feroze AH, Liu J, Kahn SA, Zhang M, et al. Disrupting the CD47-SIRP $\alpha$ anti-phagocytic axis by a humanized anti-CD47 antibody is an efficacious treatment for malignant pediatric brain tumors. Science translational medicine. 2017;9(381).

57. Liu J, Wang L, Zhao F, Tseng S, Narayanan C, Shura L, et al. Pre-Clinical Development of a Humanized Anti-CD47 Antibody with Anti-Cancer Therapeutic Potential. PloS one. 2015;10(9):e0137345.

58. Lu T, Yang $X$, Shi $Y$, Zhao M, Bi G, Liang J, et al. Single-cell transcriptome atlas of lung adenocarcinoma featured with ground glass nodules. Cell discovery. 2020;6:69.

59. Chen Z, Zhou L, Liu L, Hou Y, Xiong M, Yang Y, et al. Single-cell RNA sequencing highlights the role of inflammatory cancer-associated fibroblasts in bladder urothelial carcinoma. Nature 
communications. 2020;11(1):5077.

2 60. Guo YJ, Zhou YJ, Yang XL, Shao ZM, Ou ZL. The role and clinical significance of the CXCL17-

3 CXCR8 (GPR35) axis in breast cancer. Biochemical and biophysical research communications. 4 2017;493(3):1159-67.

5 61. Karacz CM, Yan J, Zhu H, Gerber DE. Timing, Sites, and Correlates of Lung Cancer Recurrence. 6 Clinical lung cancer. 2020;21(2):127-35.e3.

7 62. Kamigaichi A, Tsutani Y, Fujiwara M, Mimae T, Miyata Y, Okada M. Postoperative Recurrence 8 and Survival After Segmentectomy for Clinical Stage 0 or IA Lung Cancer. Clinical lung cancer. 9 2019;20(5):397-403.e1.

10 63. Gao S, Li N, Gao S, Xue Q, Ying J, Wang S, et al. Neoadjuvant PD-1 inhibitor (Sintilimab) in 11 NSCLC. Journal of thoracic oncology : official publication of the International Association for the 12 Study of Lung Cancer. 2020;15(5):816-26.

13 64. Shu CA, Gainor JF, Awad MM, Chiuzan C, Grigg CM, Pabani A, et al. Neoadjuvant 14 atezolizumab and chemotherapy in patients with resectable non-small-cell lung cancer: an open15 label, multicentre, single-arm, phase 2 trial. The Lancet Oncology. 2020.

16 65. Forde PM, Chaft JE, Smith KN, Anagnostou V, Cottrell TR, Hellmann MD, et al. Neoadjuvant PD-1 Blockade in Resectable Lung Cancer. The New England journal of medicine. 18 2018;378(21):1976-86.

19 66. Chen S, Zhou Y, Chen Y, Gu J. fastp: an ultra-fast all-in-one FASTQ preprocessor. 20 Bioinformatics (Oxford, England). 2018;34(17):i884-i90.

21 67. Marjanovic ND, Hofree M, Chan JE, Canner D, Wu K, Trakala M, et al. Emergence of a High22 Plasticity Cell State during Lung Cancer Evolution. Cancer cell. 2020;38(2):229-46.e13.

23 68. Vento-Tormo R, Efremova M, Botting RA, Turco MY, Vento-Tormo M, Meyer KB, et al. Single24 cell reconstruction of the early maternal-fetal interface in humans. Nature. 2018;563(7731):3472553.

26 69. Jin S, Guerrero-Juarez CF, Zhang L, Chang I, Myung P, Plikus MV, et al. Inference and analysis 27 of cell-cell communication using CellChat. 2020:2020.07.21.214387.

28 70. Ashburner M, Ball CA, Blake JA, Botstein D, Butler H, Cherry JM, et al. Gene ontology: tool for 29 the unification of biology. The Gene Ontology Consortium. Nature genetics. 2000;25(1):25-9. 


\begin{tabular}{|c|c|c|c|c|}
\hline \multirow{2}{*}{ Characteristics } & \multicolumn{2}{|c|}{$\begin{array}{c}\text { scRNA cohort } \\
(\mathrm{n}=31)\end{array}$} & \multicolumn{2}{|c|}{$\begin{array}{c}\text { WES/mIHC cohort } \\
(n=54)\end{array}$} \\
\hline & $\begin{array}{c}\text { AIS/MIA, n (\%) } \\
(n=16)\end{array}$ & $\begin{array}{c}\text { IAC, } n(\%) \\
(n=15)\end{array}$ & $\begin{array}{c}\text { AIS/MIA, n (\%) } \\
(n=30)\end{array}$ & $\begin{array}{c}\text { IAC, } n(\%) \\
(n=24)\end{array}$ \\
\hline \multicolumn{5}{|l|}{ Gender } \\
\hline Male & $3(18.8)$ & $10(66.7)$ & $7(23.3)$ & $11(45.8)$ \\
\hline Female & $13(81.2)$ & $5(33.3)$ & $23(76.7)$ & $13(54.2)$ \\
\hline Age & $53 \pm 11.7$ & $60 \pm 11.4$ & $49.5 \pm 11.1$ & $58.5 \pm 10.9$ \\
\hline \multicolumn{5}{|l|}{ Smoking status } \\
\hline Non-smokers & $16(100)$ & $11(73.3)$ & $27(90.0)$ & 20 (83.3) \\
\hline $\begin{array}{l}\text { Former/current } \\
\text { smokers }\end{array}$ & $0(0)$ & $4(26.7)$ & $3(10.0)$ & $4(16.7)$ \\
\hline \multicolumn{5}{|l|}{ Family history } \\
\hline Yes & $2(12.5)$ & $3(20.0)$ & $3(10.0)$ & $2(8.3)$ \\
\hline No & $14(87.5)$ & $12(80.0)$ & $27(90.0)$ & $22(91.7)$ \\
\hline Tumor size (mm) & $8 \pm 5.4$ & $15 \pm 4.9$ & $8 \pm 4.4$ & $14.5 \pm 5.4$ \\
\hline \multicolumn{5}{|l|}{ Radiological pattern } \\
\hline pGGO & $13(81.2)$ & $3(20.0)$ & $23(76.7)$ & $5(20.8)$ \\
\hline mGGO & $3(18.8)$ & $12(80.0)$ & $7(23.3)$ & $19(79.2)$ \\
\hline \multicolumn{5}{|l|}{ Type of surgery } \\
\hline Wedge resection & $7(43.75)$ & $3(20.0)$ & $15(50.0)$ & $5(20.8)$ \\
\hline Segmentectomy & $6(37.5)$ & $6(40.0)$ & $9(30.0)$ & $10(41.7)$ \\
\hline Lobectomy & $3(18.75)$ & $6(40.0)$ & $6(20.0)$ & $9(37.5)$ \\
\hline \multicolumn{5}{|l|}{ Pleural invasion } \\
\hline Positive & $0(0)$ & $2(13.3)$ & $0(0)$ & $2(8.3)$ \\
\hline Negative & $16(100)$ & $13(86.7)$ & $30(100)$ & $22(91.7)$ \\
\hline \multicolumn{5}{|l|}{ EGFR status } \\
\hline Mutation & $6(37.5)$ & $11(73.3)$ & $8(26.7)$ & $16(66.7)$ \\
\hline Wild type & $10(62.5)$ & $4(26.7)$ & $22(73.3)$ & $8(33.3)$ \\
\hline \multicolumn{5}{|l|}{ PD-L1 (Dako 22C3) } \\
\hline$<1 \%$ & $15(93.75)$ & $10(66.7)$ & $27(90.0)$ & 20 (83.3) \\
\hline$\geqslant 1 \%$ & $1(6.25)$ & $5(33.3)$ & $3(10.0)$ & $4(16.7)$ \\
\hline
\end{tabular}

3

4

5

6

7

8 


\section{Figures and Figure Legends}
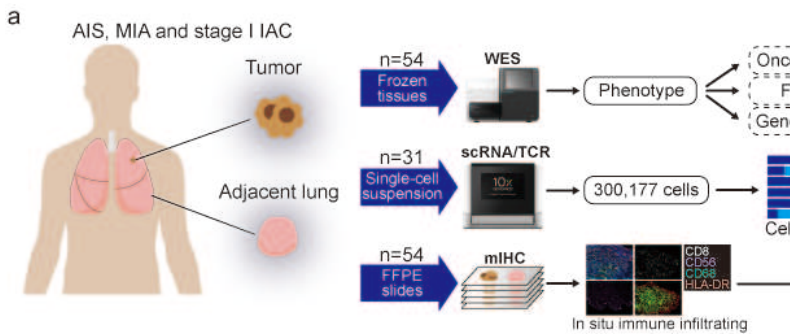

Oncogene altertion (eg. EGFR)
magn=z=n
Focal/Chromosome CNV

Integrated Stratified Analysis

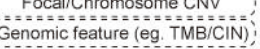

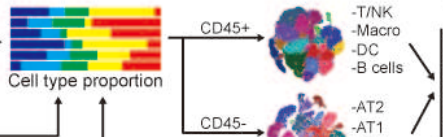
Adjacent Differential Expression Function Enrichment

External Normal Control Datasets $(n=8)$

Normal Lung Dona

Number of cells
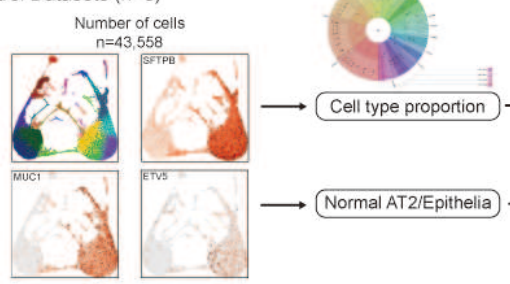

Lineage Evolution
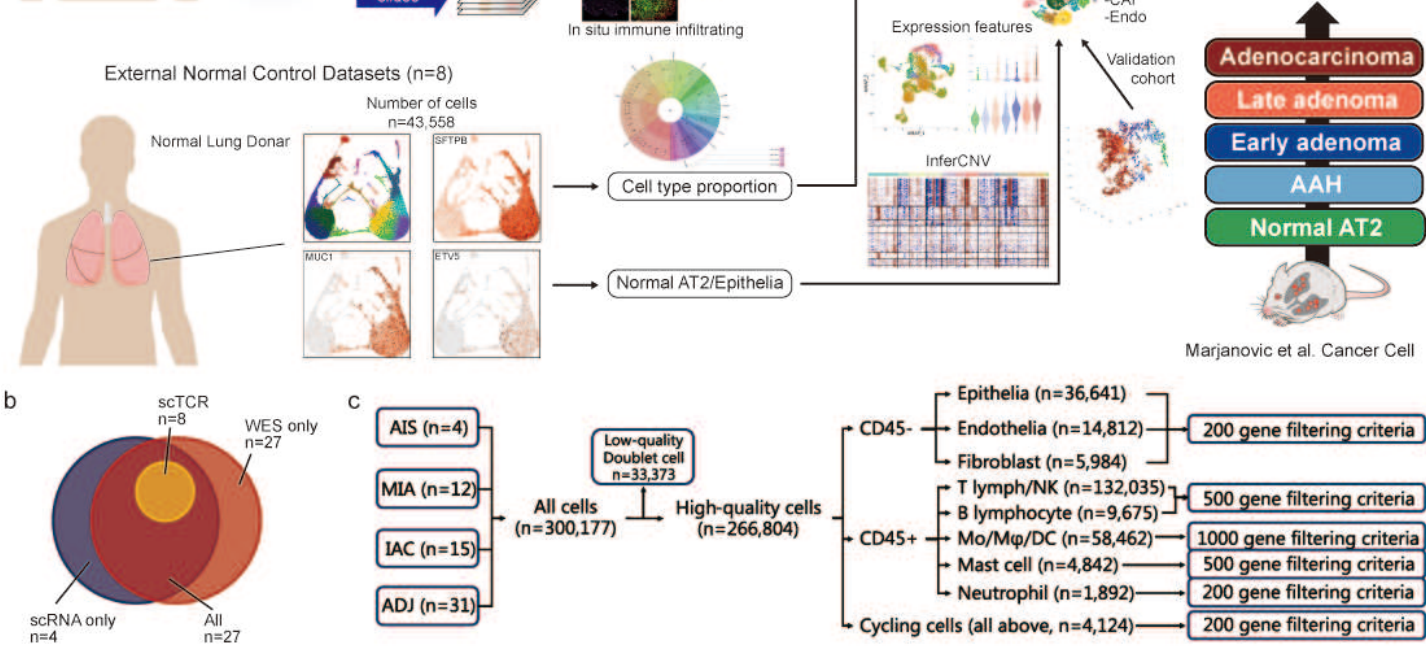

$\uparrow$ Validation

Adenocarcinoma
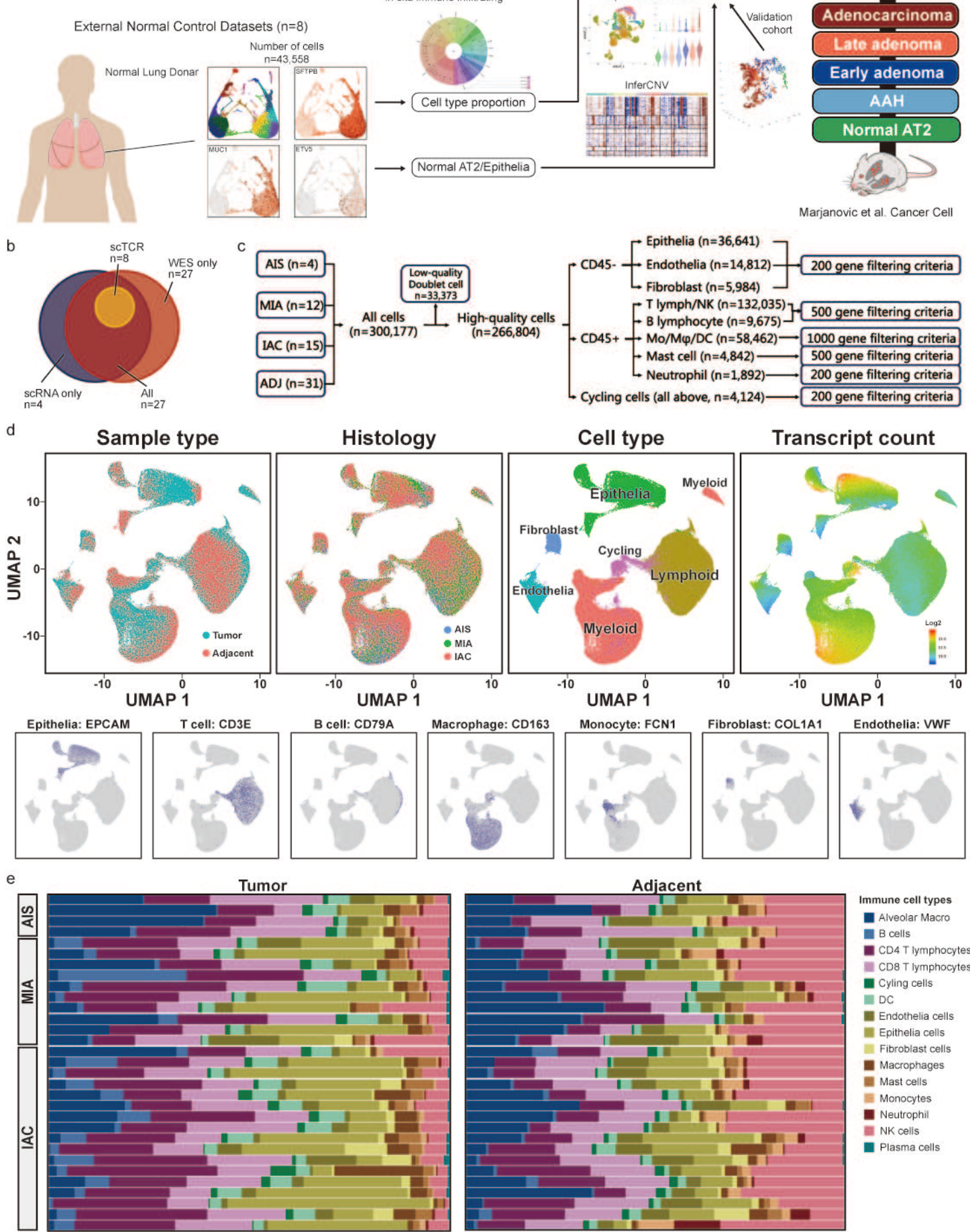

Immune cell types - Alveolar Macr B cells

CD4 T lymphocytes CD8 Tlymphoc DC

Endothelia cells Endothelia cells - Ephria cells - Mroblast cells Macrophages Mast cells Monocytes - Neutrophi In NK cells - Plasma cells

Figure 1. Immune infiltrating microenvironment in precursor lesions and stage I lung adenocarcinoma. a, Research roadmap of the overall study. Two major cohorts were involved in 
1 the current study including pre-/invasive lung adenocarcinoma (for single-cell RNA sequencing,

$2 \mathrm{n}=31$; for whole exome sequencing/multiplex immunohistochemistry, $\mathrm{n}=54$ ) and normal control

3 donors (single-cell RNA sequencing, $n=8$ ). Each enrolled patient for scRNA has at least one pair

4 tissue containing tumor specimens and adjacent lung tissues. Sequential analytic strategies

5 following multi-omics data were summarized in the scheme. AIS, adenocarcinoma in situ; MIA,

6 minimally invasive adenocarcinoma; IAC, invasive adenocarcinoma; WES, whole exome

7 sequencing; scRNA, single-cell RNA sequencing; mIHC, multiplex immunohistochemistry; TCR,

8 T cell receptor repertoire. b, Sample distribution among different omics sequencing. c, Flow chart

9 of enrolled samples and filtering cells for subsequent analysis. d, Cell distribution regarding

10 sample type, histology, cell types and transcriptome counts were summarized along with featured markers of different cell types. e, Proportion of different cell types between tumor specimens and adjacent lung tissues stratified by histology and specific mutational profile. Each row represents one patient.
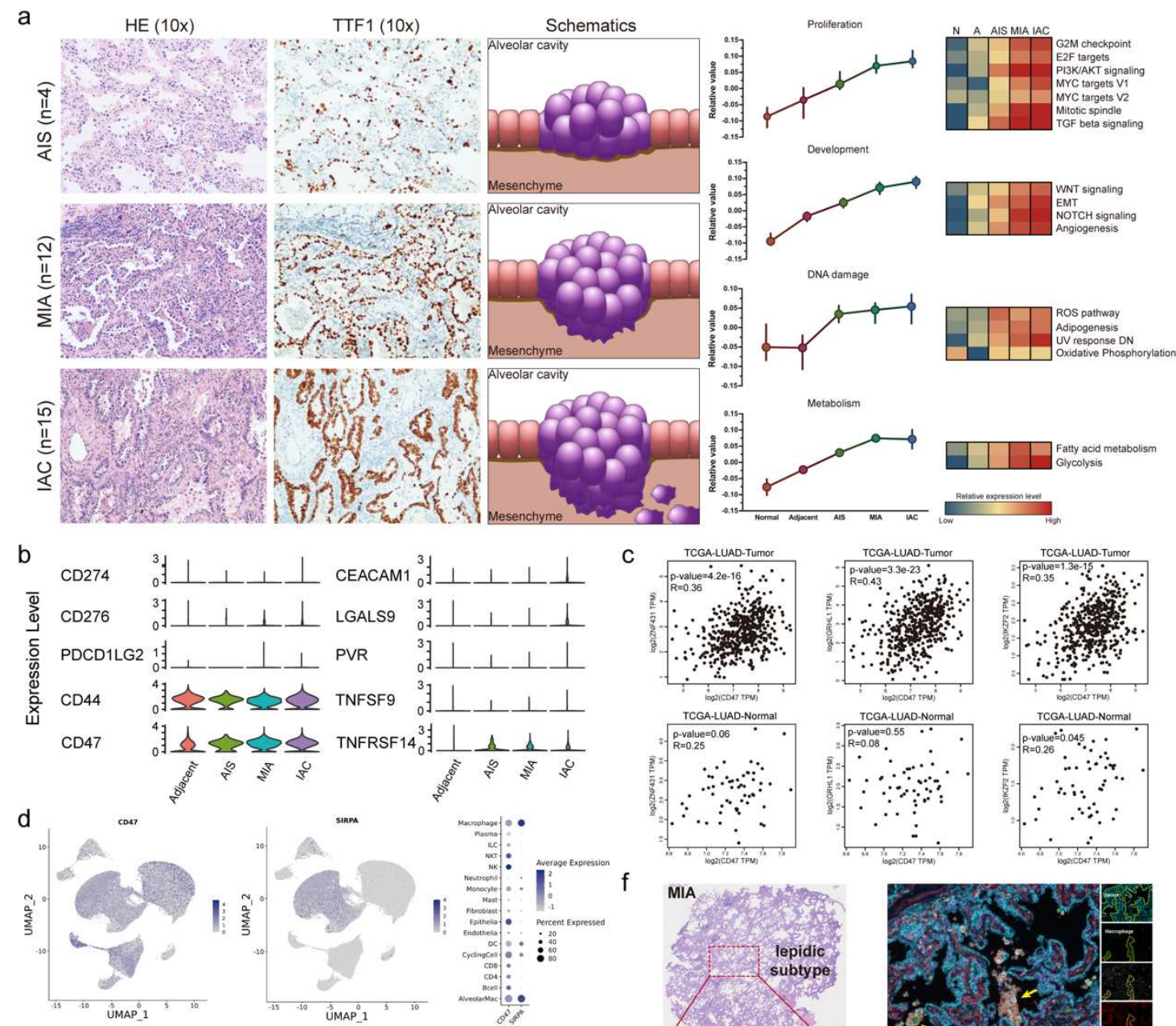

e

C
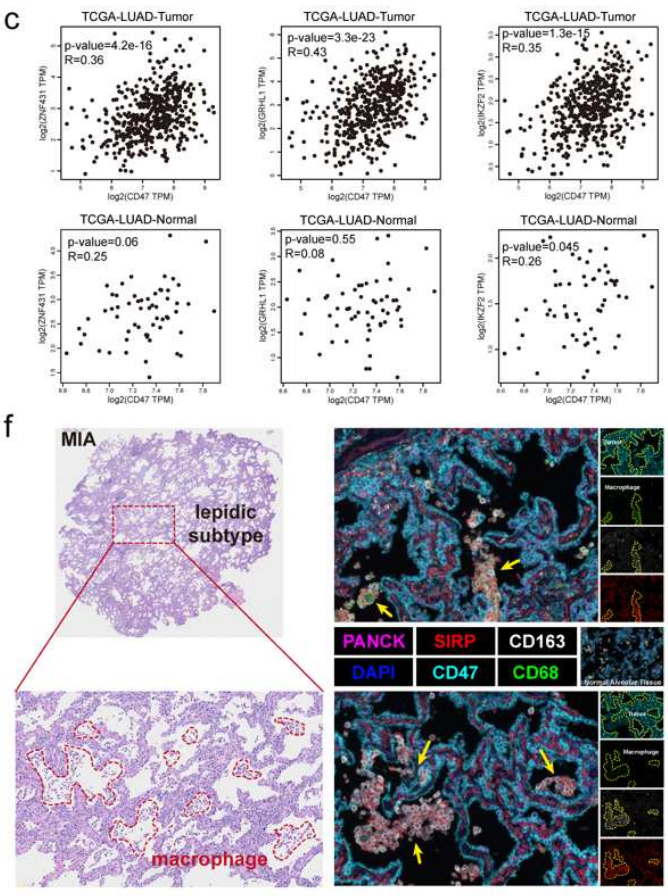

Figure 2. Cancer hallmark features and potential immune targets for preinvasive and early 
invasive LUAD.

a, Hallmark features of different developmental stages in tumor cells. Matched HE staining along with TTF-1 staining slides were presented. Relative expression level of cell proliferation, development, DNA damage repair and metabolism were analyzed regarding normal lung tissue ("N"), adjacent tissue ("A"), AIS, MIA and IAC. AIS, adenocarcinoma in situ; MIA, minimally invasive adenocarcinoma; IAC, invasive adenocarcinoma. b, Violin plots of immune checkpoint expression on epithelia cells upon different histology subtypes. c, Correlative expression of CD47 and selected genes through WGCNA in LUAD and corresponding normal lung tissues. d, Expression of CD47 and SIRPA shown in UMAP and bubble plots of detailed expression level regarding different cell types. e, Expression of CD47 in genetically engineered mouse model regarding different developmental status of lung adenocarcinoma. t-test was performed independently to evaluate significance between different stages. f, Representative multiplex fluorescence of a MIA showing distribution of macrophage and tumor cells. The panel includes PANCK, DAPI, CD47, SIRP, CD163 and CD68 in corresponding color. Regions of infiltrating macrophages were circled and pointed by yellow dotted lines arrows, respectively.

a
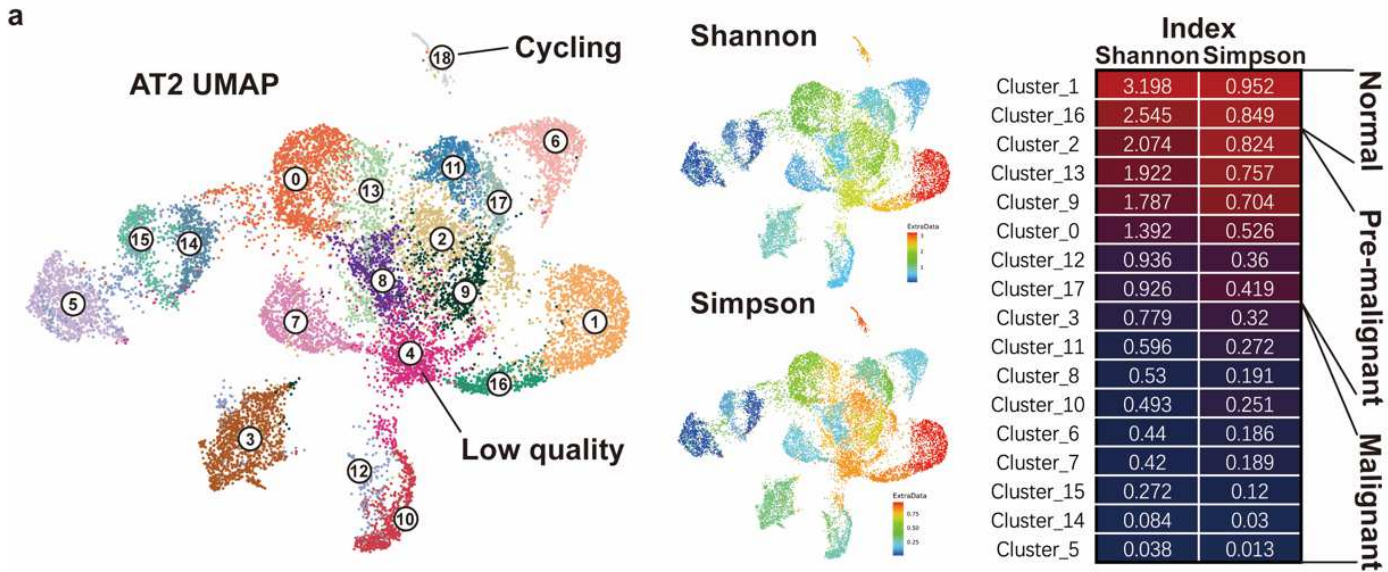

b
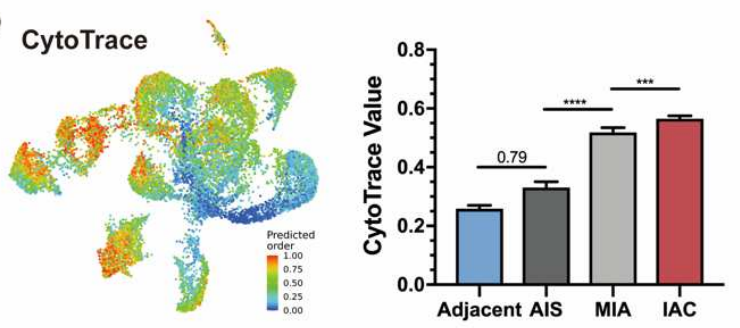

C
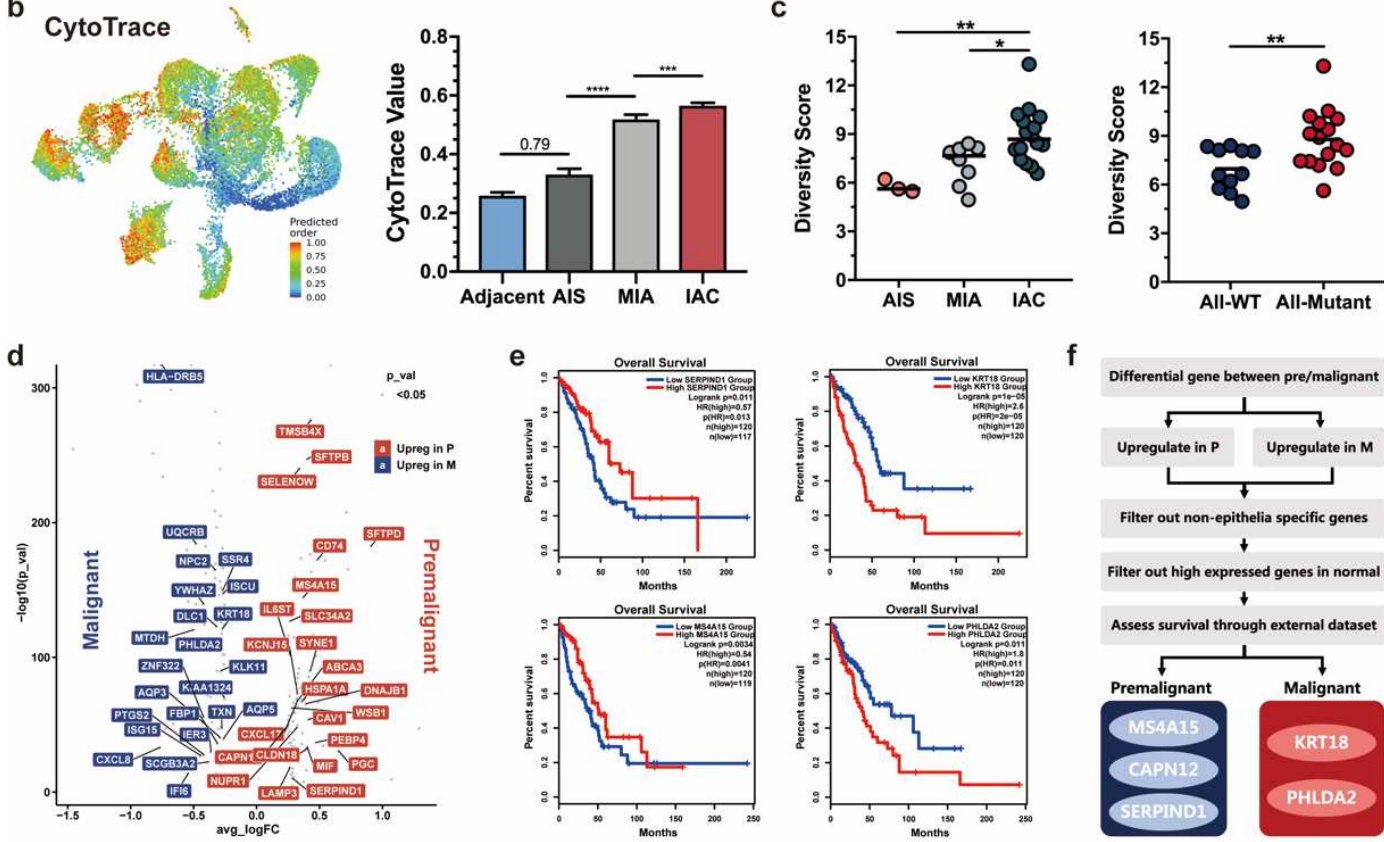

Figure 3. Intercellular heterogeneity and differentiation of AT2 for preinvasive and early 
2 a, Different cluster annotation of AT2 cell type through UMAP. Clusters of AT2 were classified 3 into normal, pre-malignant and malignant group regarding Shannon and Simpson index. AT2, 4 alveolar type II. b, Intercellular heterogeneity (ITH) of AT2 cells through CytoTrace. CytoTrace 5 values were measured in different histologic stages. t-test was used independently to evaluate differences between groups. c, Diversity score of AT2 cells regarding stratified by histology subtypes and EGFR mutation status. d, Volcano plot of differentially expressed genes between pre-malignant and malignant group. Genes of significance were highlighted for both phenotypes. e, Survival analysis of candidate genes through TCGA datasets. f, Flowchart of genes specifically

a

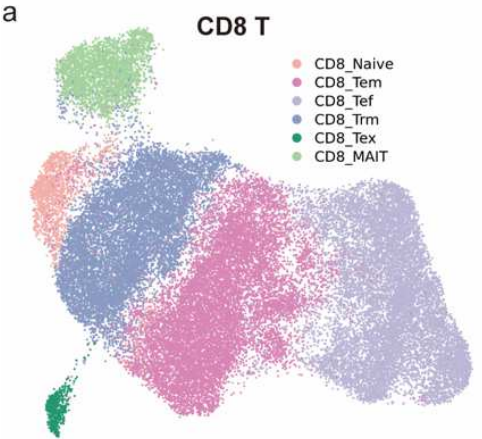

C

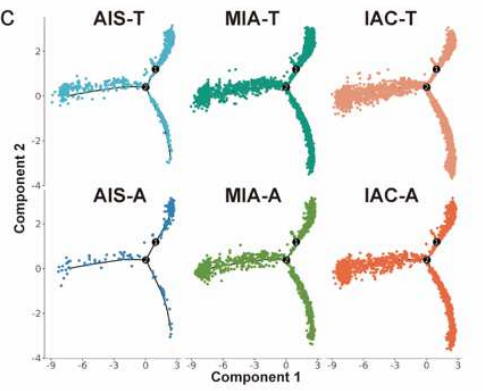

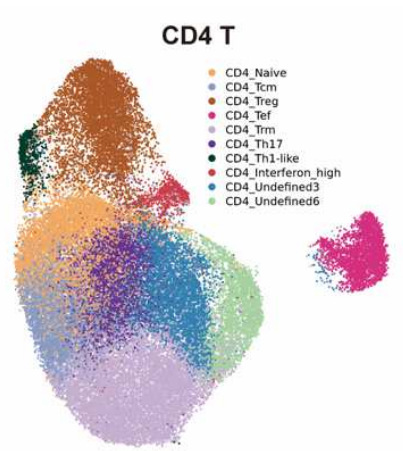
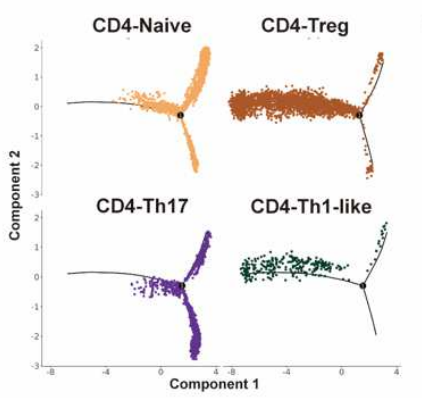

b $\frac{\mathrm{CD} 3+\mathrm{CD} 4+}{\mathrm{CD} 3+\mathrm{CD} 8+}$

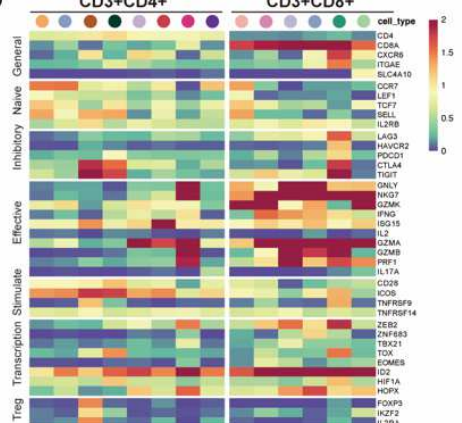

d
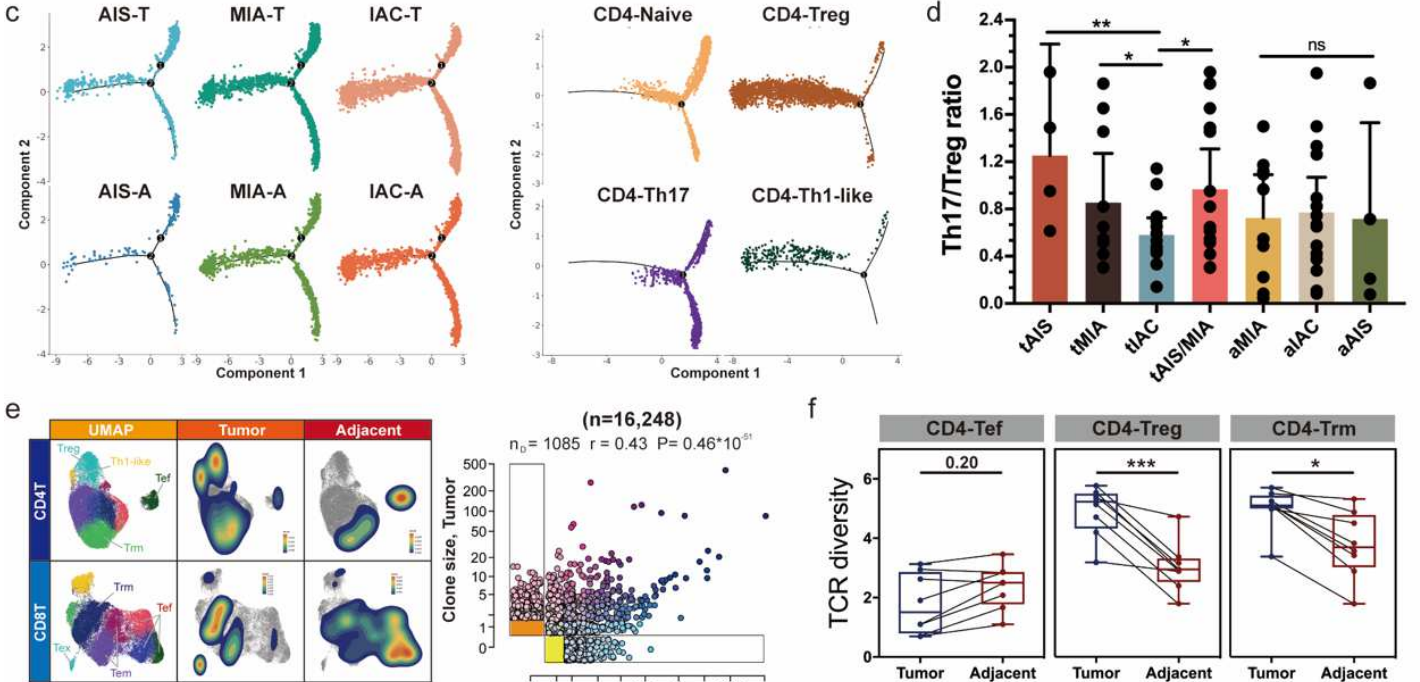

$(n=16,248)$
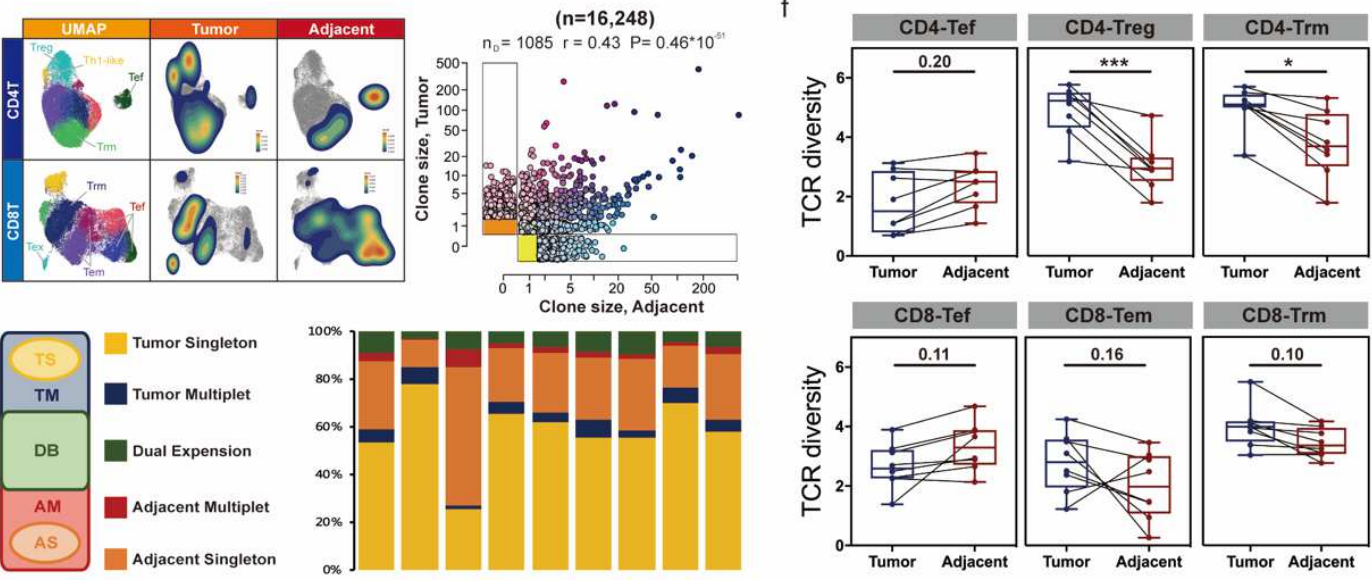

Figure 4. Landscape of $T$ lymphocytes and imbalance of Th17/Treg triggers disease progression.

a, UMAP of CD4 and CD8 T lymphocyte populations with specific annotation. b, Heatmap of featured genes among different subtypes of CD4 and CD8 lymphocytes. c, Pseudo-time of CD4 T lymphocytes regarding sample types and cell types. d, Th17/Treg ratio of tumor and adjacent tissue among different histology subtypes. One-way ANOVA and t-test were applied to measure 
significance between groups. e, Single-cell RNA and TCR sequencing of T lymphocytes. TCR expansion level of different cell subsets were shown in colored intensity regarding sample type through scatter plots. Dots are colored by a palette for the tissue expansion. $r$ denotes Pearson's correlation coefficient, on the dual-expanded clones $\left(\mathrm{n}_{\mathrm{D}}\right)$. P values were determined by a twotailed linear regression t-test. Bar plots represent distribution of different TCR expansion pattern among patients. f, Comparison of TCR diversity between tumor and adjacent tissue for different T lymphocyte subtypes. T-test was used to evaluate the significance.

a

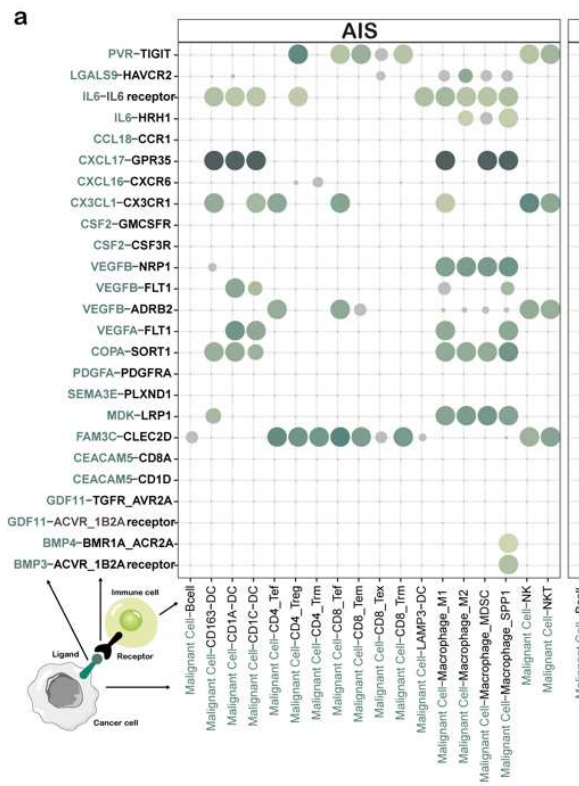

b
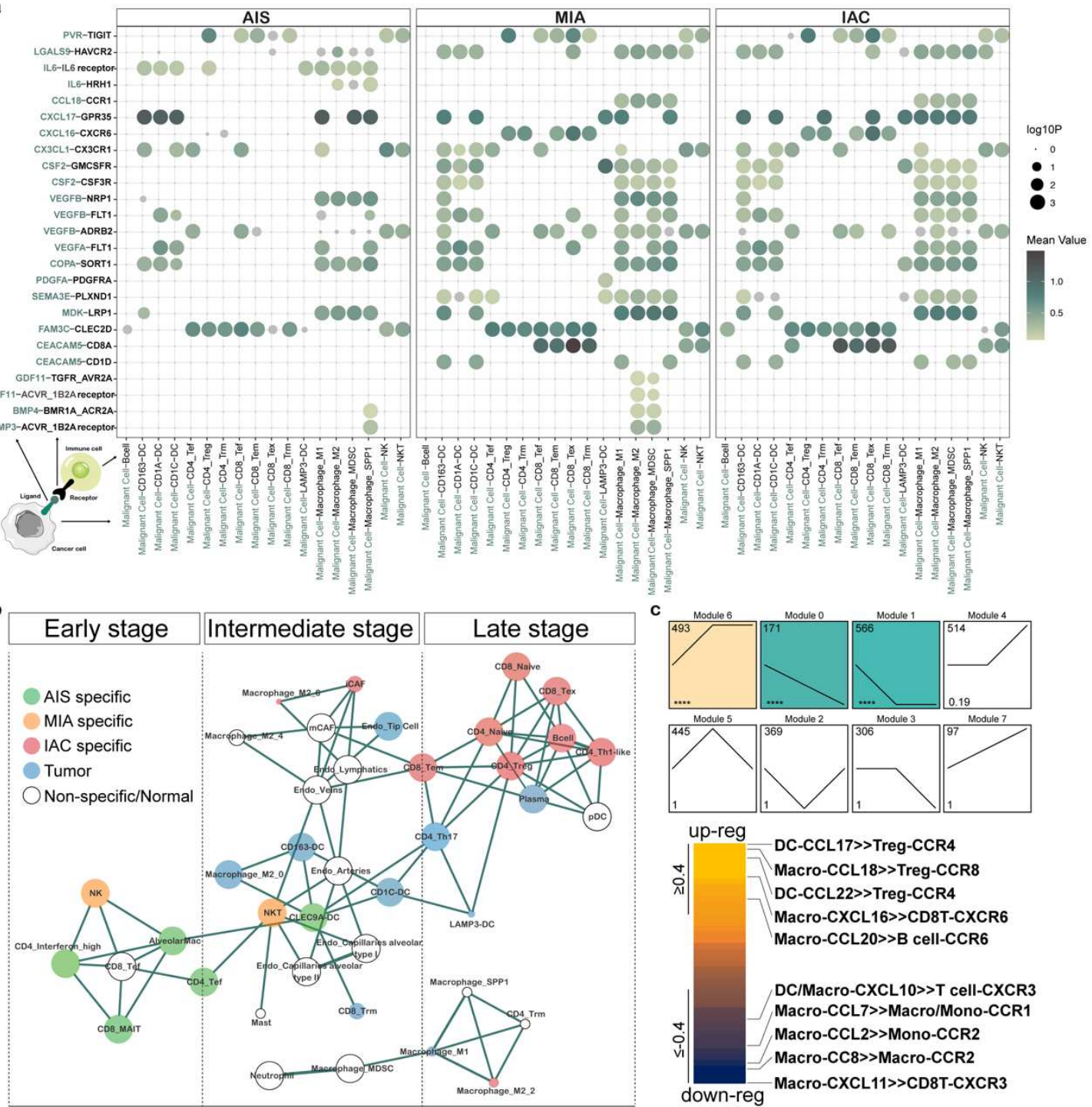

Figure 5. Cell communications revealed featured immune architecture upon stages.

a, Interaction of ligands (x axis) and receptors (y axis) between cancer cells and immune cells, respectively, through Cellphone DB. b. Comprehensive network analysis of different immune cells among sample types and histological subtypes. c, Diverse immunoregulatory modules among histological subtypes (initiative, intermediate and terminal point of the broken line represent different histological subtypes in order) and significantly up- or down regulated ligand-receptor pairs among different immune cells. 

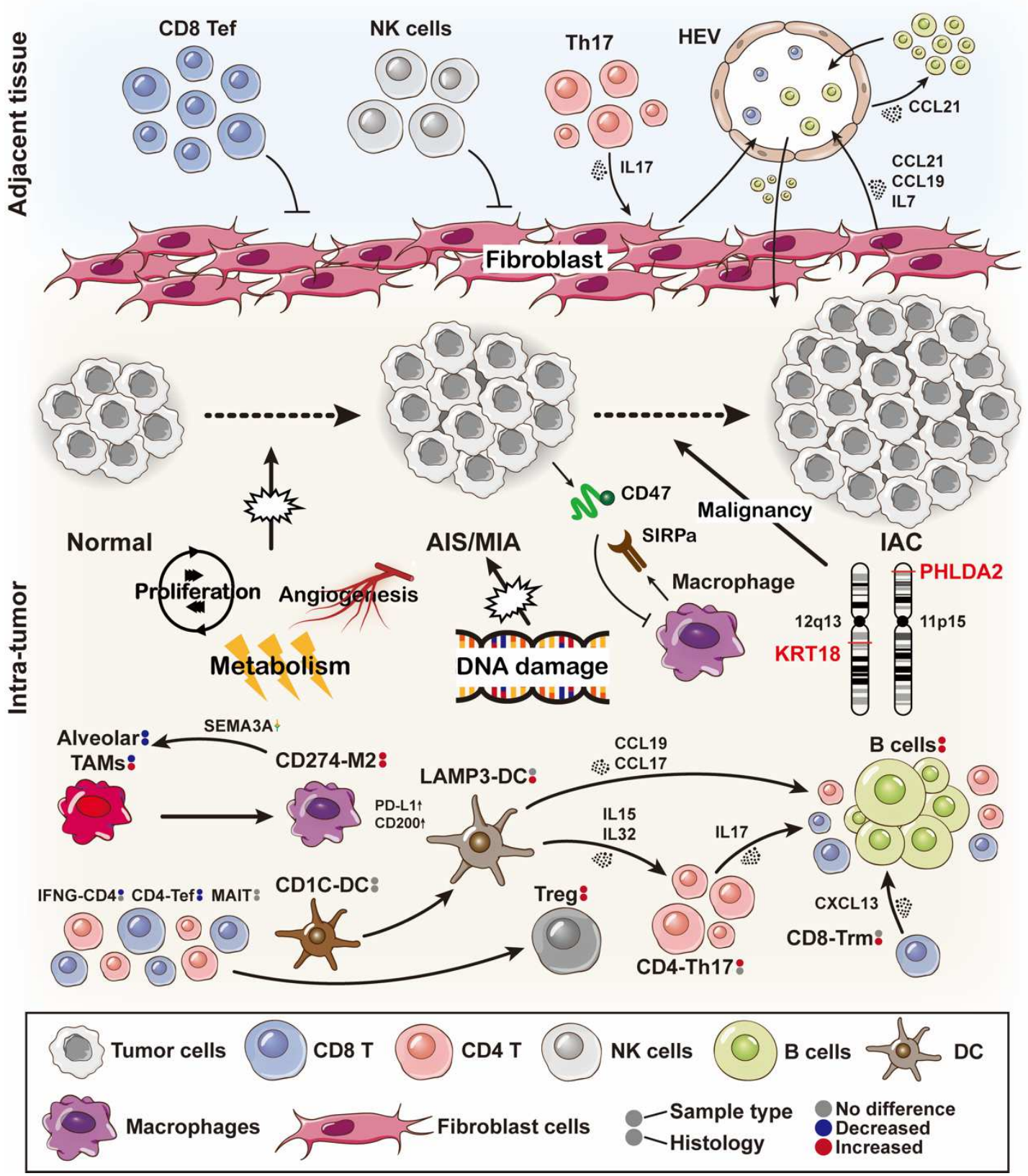

Figure 6. Comprehensive schematic model integrating intrinsic and extrinsic microenvironment during tumor development.

Featured molecular events and modulation of immune microenvironment during preinvasive LUAD development were summarized. 
1 Supplementary Figures and Figure Legends
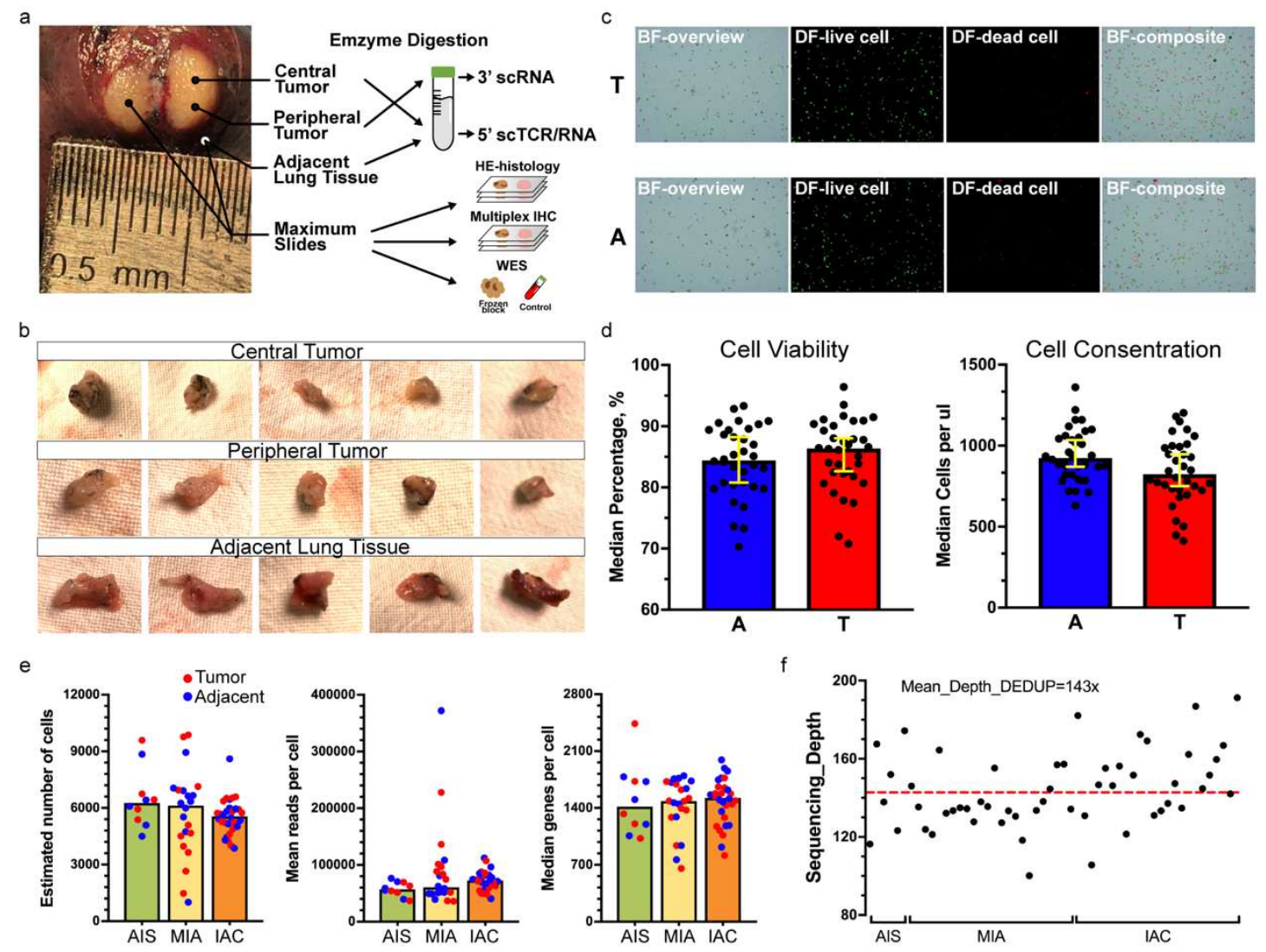

Suppl-1. Management of samples for multi-omics analyses. a. Clinical samples were bisected intraoperatively. Half of it was prepared for single-cell sequencing while the other half was stored in liquid nitrogen for whole exome sequencing and parrffin block for immunohistochemistry, respectively. For tumor single-cell suspension, we collected both central and peripheral tumor of which the longest diameter was over $3 \mathrm{~mm}$. Then, we used collagenase I+IV to digest both tissues, respectively and mixed them before further management. $\mathbf{b}$, Sampling of surgical resected tissues among central tumor, peripheral tumor and adjacent lung tissue. c, Quality control of prepared single-cell suspension. We used Countstar (ALIT Life Science Co., Ltd, China) calculate cell viability and concentration for quality control. Qualified criteria were described in Methods. BF, bright field; DF, dark field. d, Cell viability and concentration were measured by comparing tumor specimens and adjacent lung tissues. e, Summary of single-cell sequencing data regarding different histology. Red spots represent samples derived from tumor while blue spots for adjacent lung tissue. f, Mean sequencing depth after deduplication of all enrolled samples for WES. The mean sequencing depth was $143 x$. 


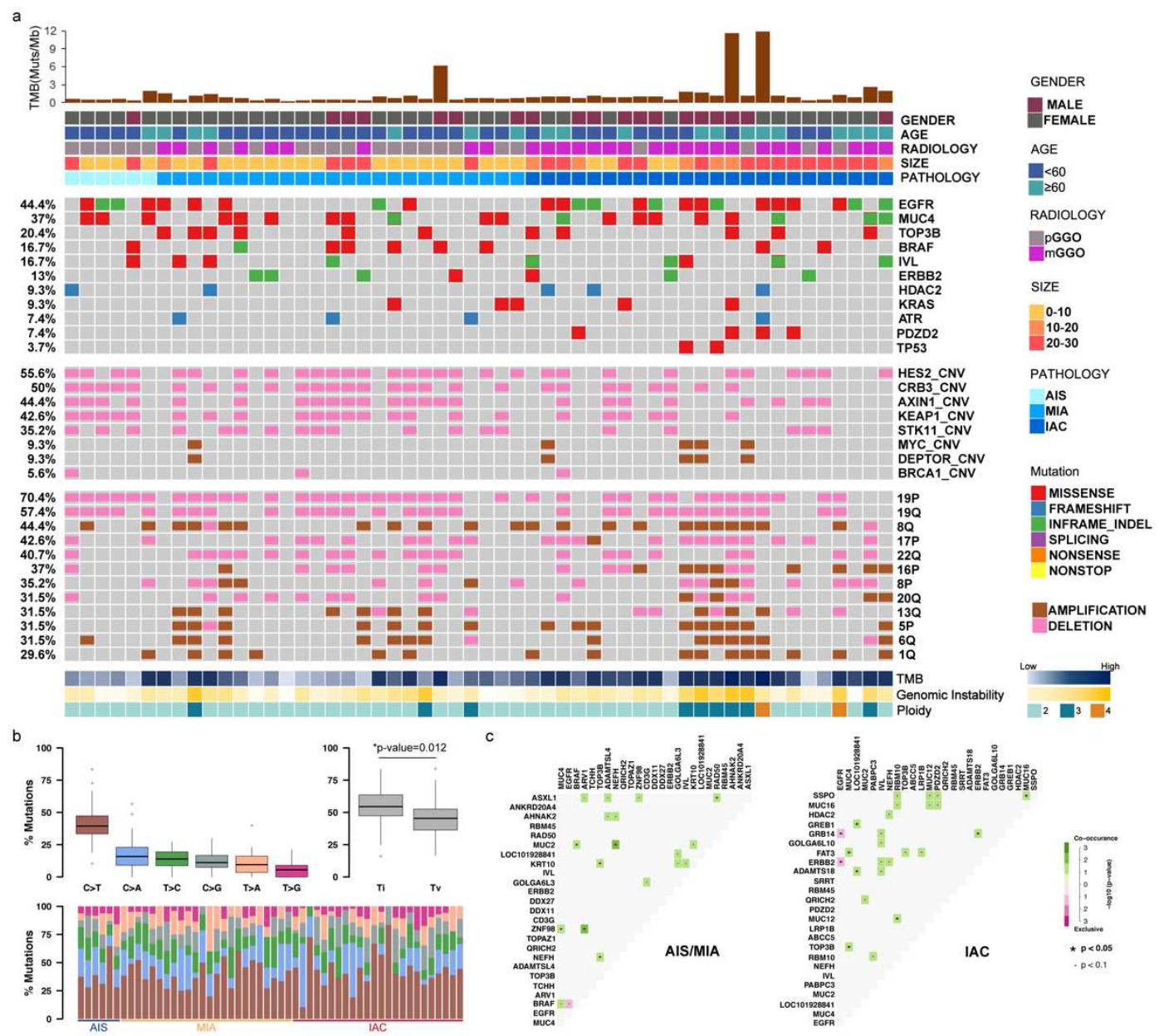

Suppl-2. Genomic characteristics of pre-invasive and invasive lung adenocarcinoma. a,

3 Comprehensive oncoprint including somatic mutations, focal and chromosome-level copy number

4 variants along with clinicopathological data was summarized. b, overall mutational spectrum of

5 early-stage lung adenocarcinoma. Transition of $\mathrm{C}>\mathrm{T}$ was the most frequent single nucleotide

6 polymorphism (SNP) pattern among early-stage lung adenocarcinoma. Overall proportion of base

7 transition was significantly higher than that of base transversion. c, Somatic interactions of

8 AIS/MIA and IAC. For each cell, the darker the green, the higher extent of co-occurrence while

9 opposite for exclusiveness. T-test was performed to calculate correlative significance between

10 genes. 

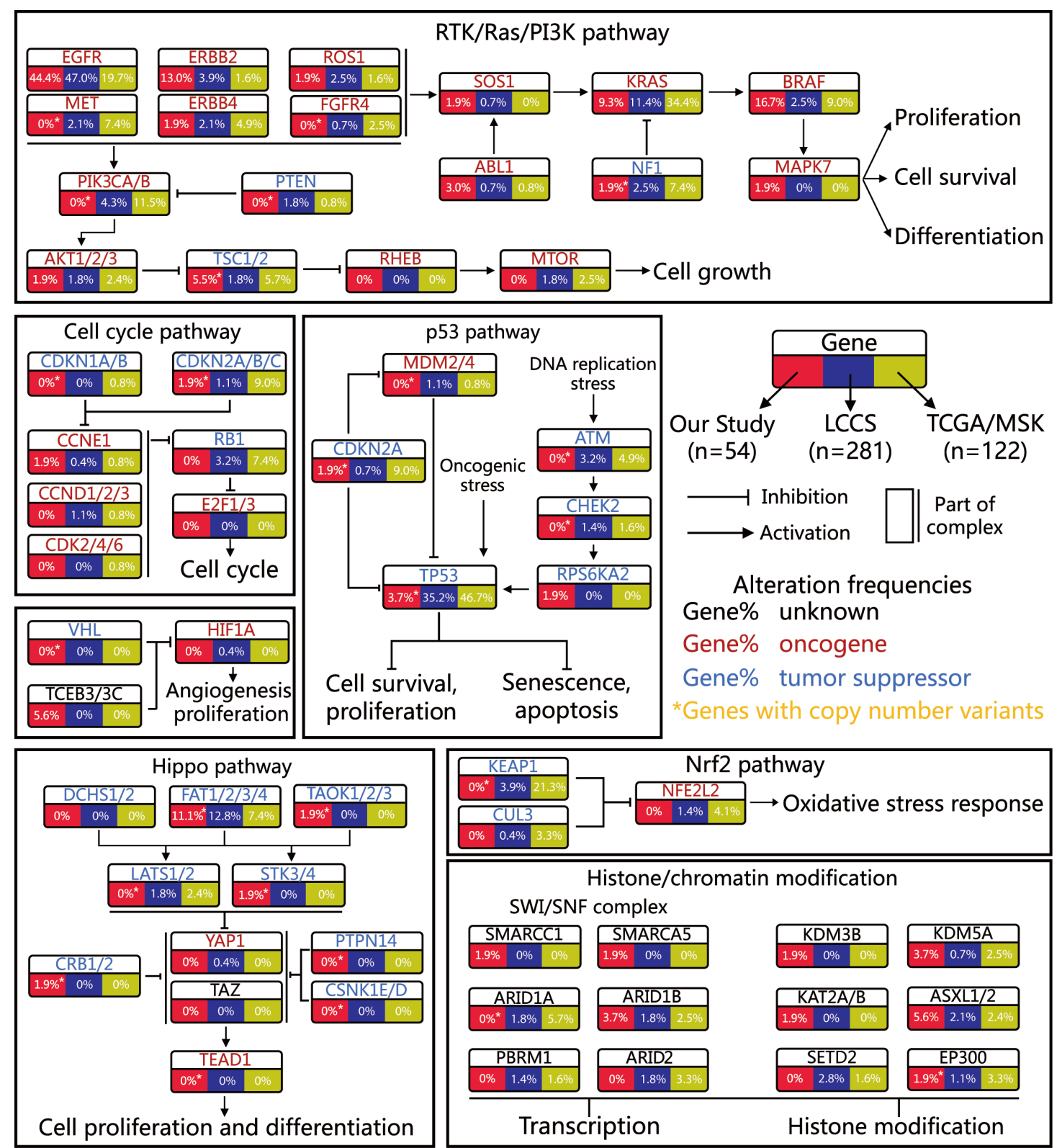

2 Suppl-3. Mutational frequencies regarding different pathway gene sets among three

3 independent cohorts. Three independent cohorts including our study ( $\mathrm{n}=54)$, East Asian lung adenocarcinomas (EAS LUADs) $(\mathrm{n}=281)$ and TCGA/MSKCC datasets $(\mathrm{n}=122)$. All enrolled data were pathologically diagnosed as lung adenocarcinoma without stage IV disease. Both EAS LUADs and TCGA/MSKCC datasets contained stage I-III invasive lung adenocarcinoma. Frequencies of genes correlated with RTK/Ras/PI3K pathway, p53 pathway, cell cycle pathway, angiogenesis pathway, hippo pathway, Nrf2 pathway and histone/ chromatin modification pathway were calculated and shown following rules presented in the figure. 


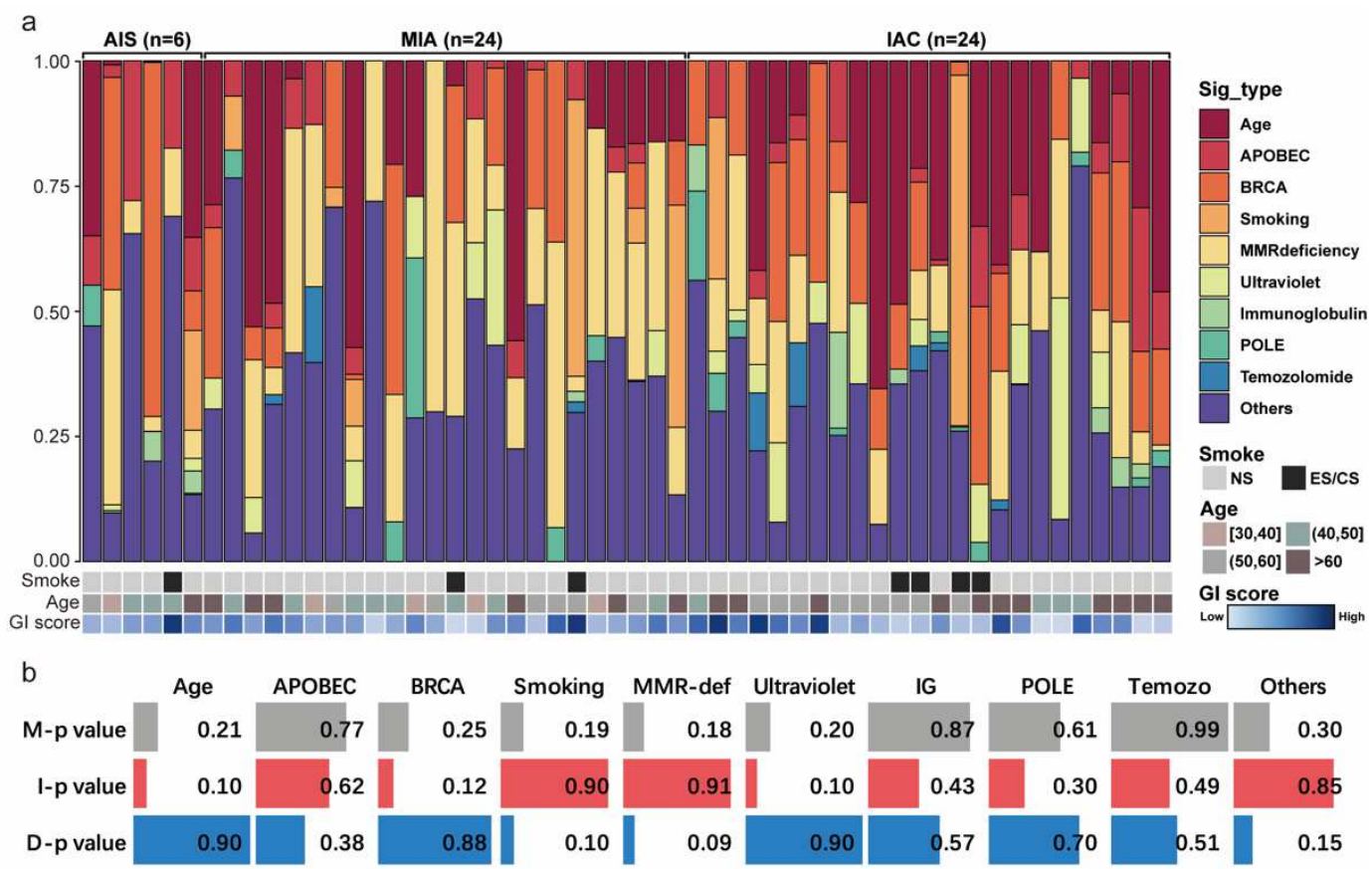

c

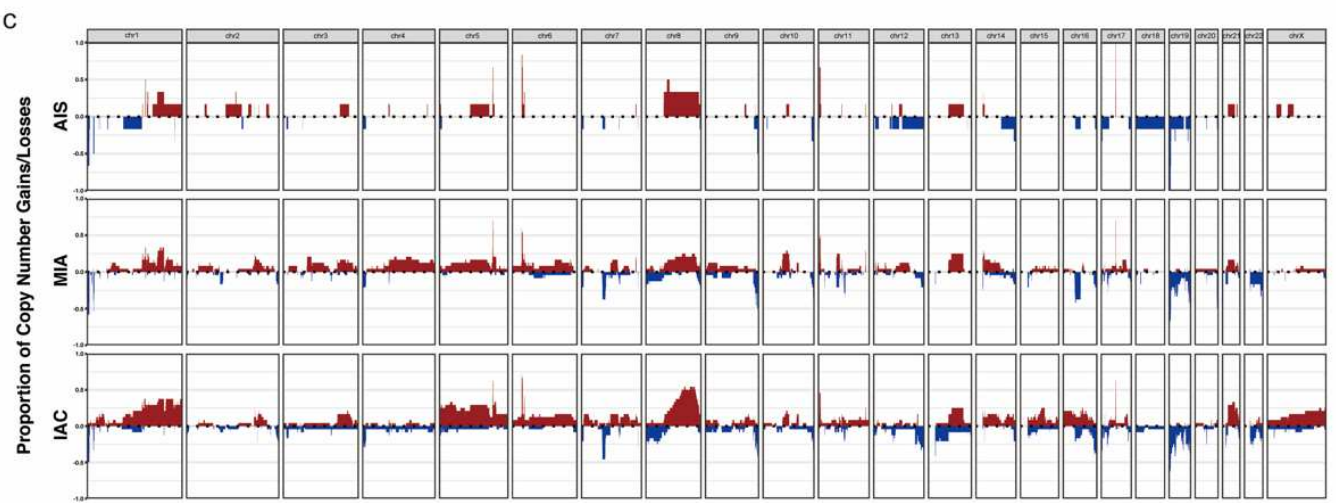

Suppl-4. Genomic disparities regarding different pathological stage of lung adenocarcinoma.

a, Differential distribution of mutational signatures among AIS, MIA and IAC and correlation with several clinicopathological variables. NS, non-smoker; ES/CS, ever-smoker/current smoker; GI, genomic instability. b, Measurement of monotonic p-value, increasing p-value and decreasing $p$-value among different mutational signatures upon different pathology. M-p value, monotonic $p$ value; I-p value, increasing p-value; D-p value, decreasing p-value; IG, immunoglobulin. c, Proportion of arm-level copy number gain/loss among different pathology. Red bar represents copy number gain while copy number loss for blue bar. Cut-off value for copy number gain and loss was 2.3 and 1.74 , respectively. 
a
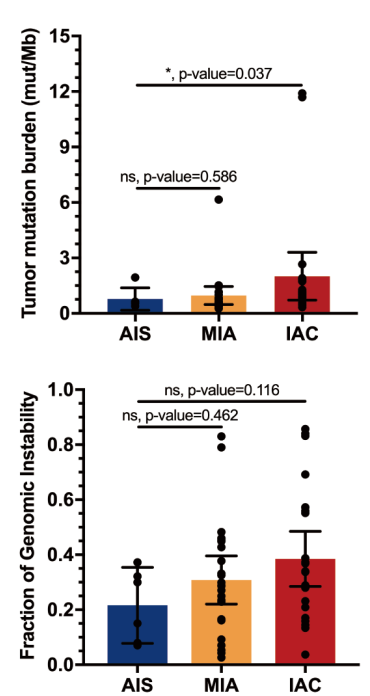

b
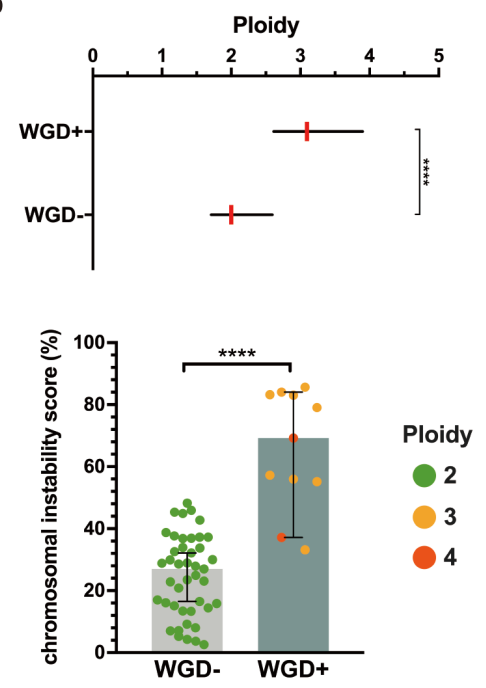
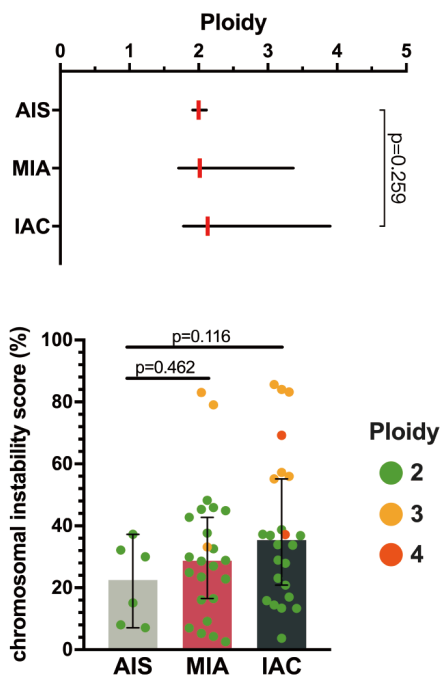

\section{Supp1-5. Prevalence of genomic characteristics among different pathology. a, Bar plots of}

tumor mutation burden (TMB) and genomic instability among AIS, MIA and IAC. Statistical significance was calculated under two-sided Mann-Whitney U test. b. Correlation of tumor ploidy, whole genome doubling and different pathology. Statistical significance was determined through one-way analysis of variance (ANOVA) for right lower plot while two-sided Mann-Whitney U test for others. 


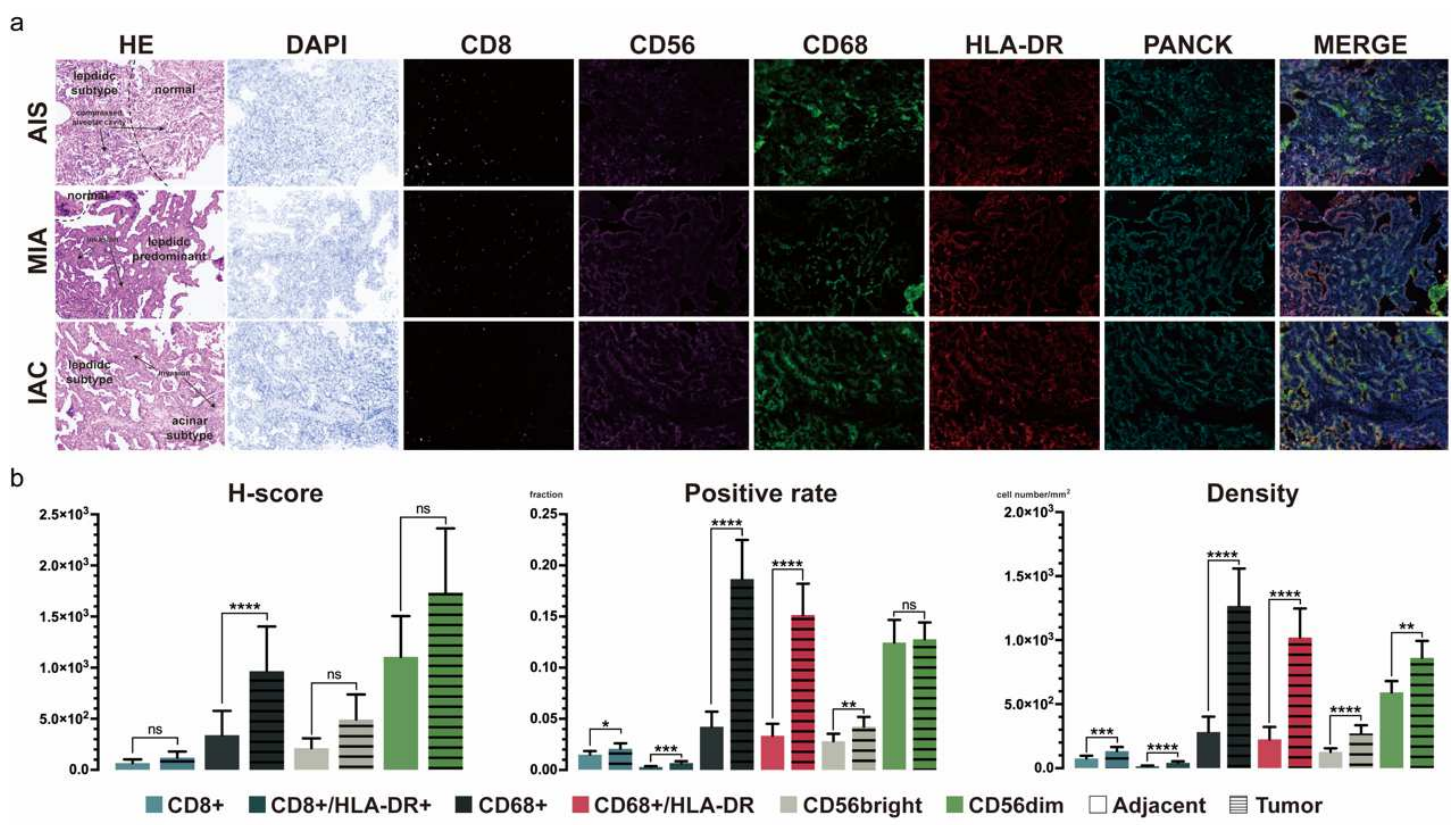

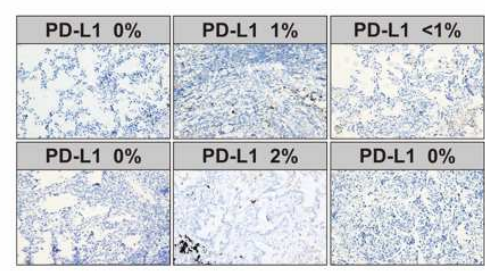

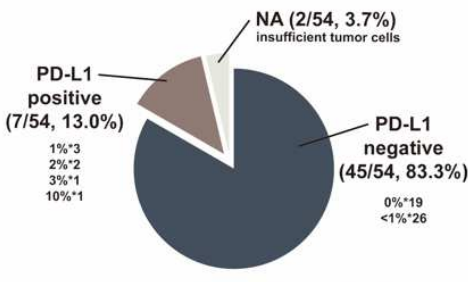

PD-L1 expression (Dako 22c3) d

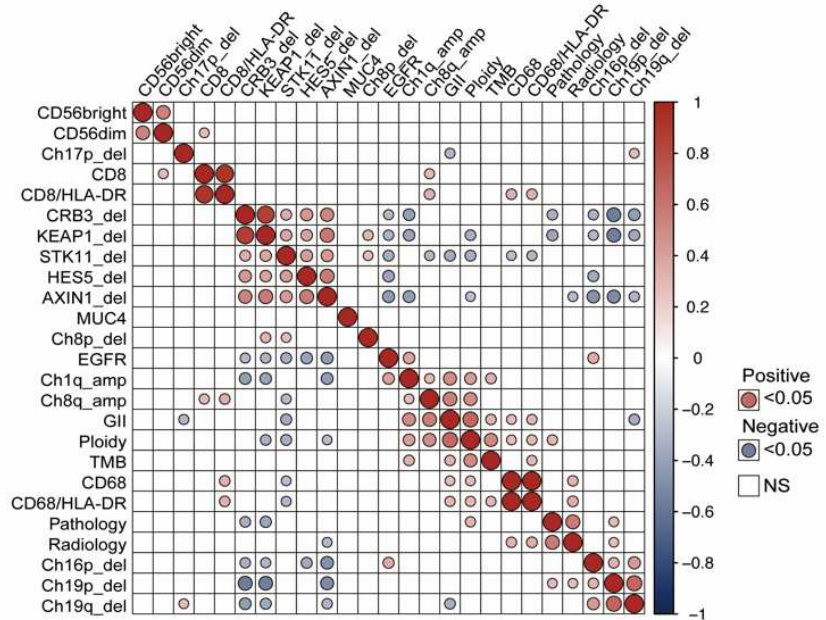

Suppl-6. Infiltrating Immune microenvironment through multiplex immunohistochemistry (mIHC) and correlation with genomic features. a, representative mIHC staining slides for different pathological stages. Arrow and annotation were presented to clarify regions of different subtypes and normal tissue. b, H-score, positive rate and density of CD8, CD56, CD68 along with HLA-DR were shown through bar plots and statistical comparison was performed through t-test. c, Evaluation of PD-L1 using Dako 22C3 assay among different pathological stages and proportion of different PD-L1 status were shown in pie chart. d, Heatmap of correlation between infiltrating immune features and genomic features including genomic instability, tumor mutation burden, ploidy and other focal or chromosomal alteration. GII, genomics instability; TMB, tumor mutation burden. 


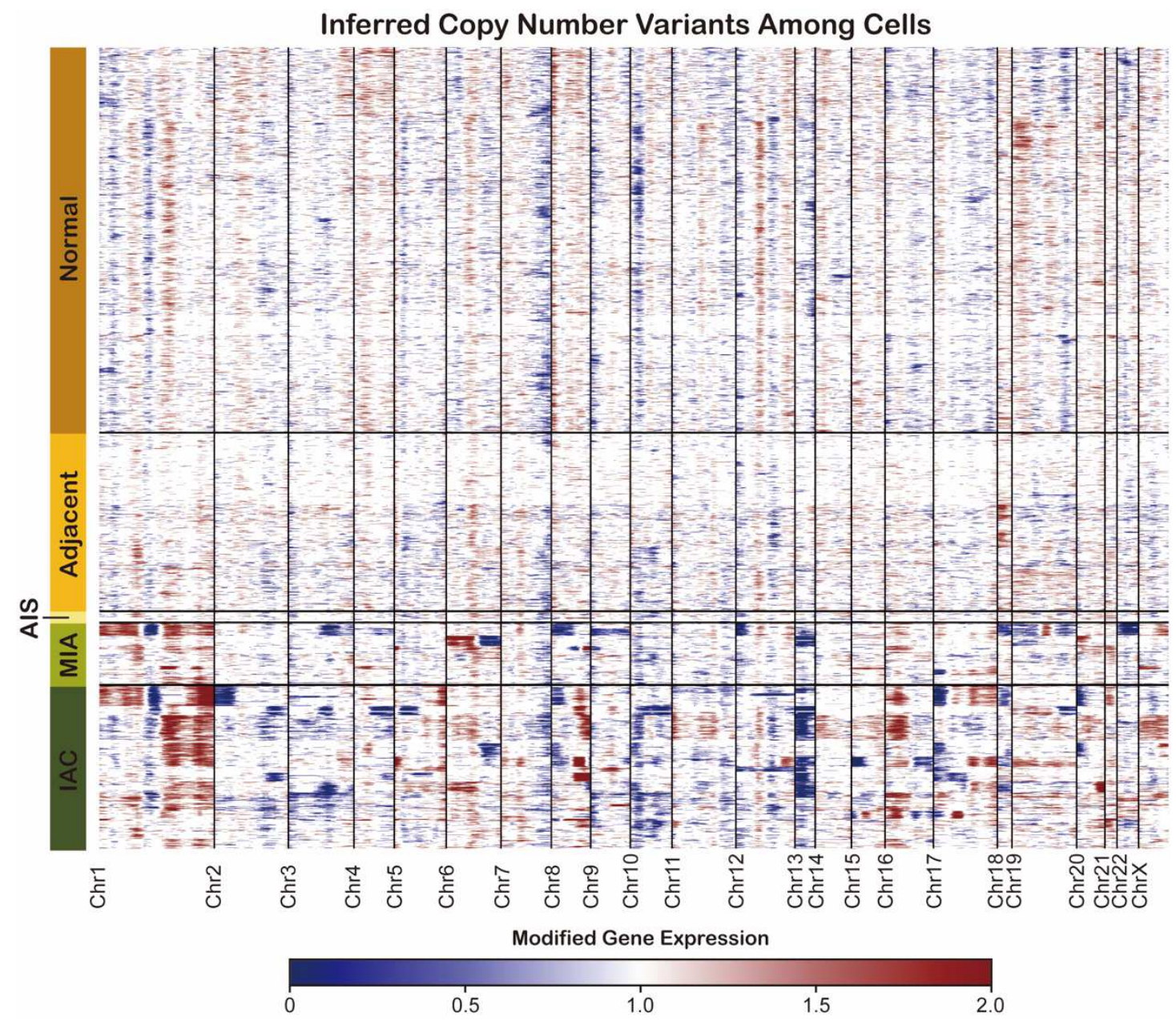

Suppl-7. Malignant tumor cells defined by inferred copy number variants for single-cell

3 sequencing. Abnormal alteration in chromosomes among normal tissue, adjacent tissue, AIS, MIA and IAC were presented with red bar indicating chromosomal amplification and blue bar 5 indicating chromosomal deletion.

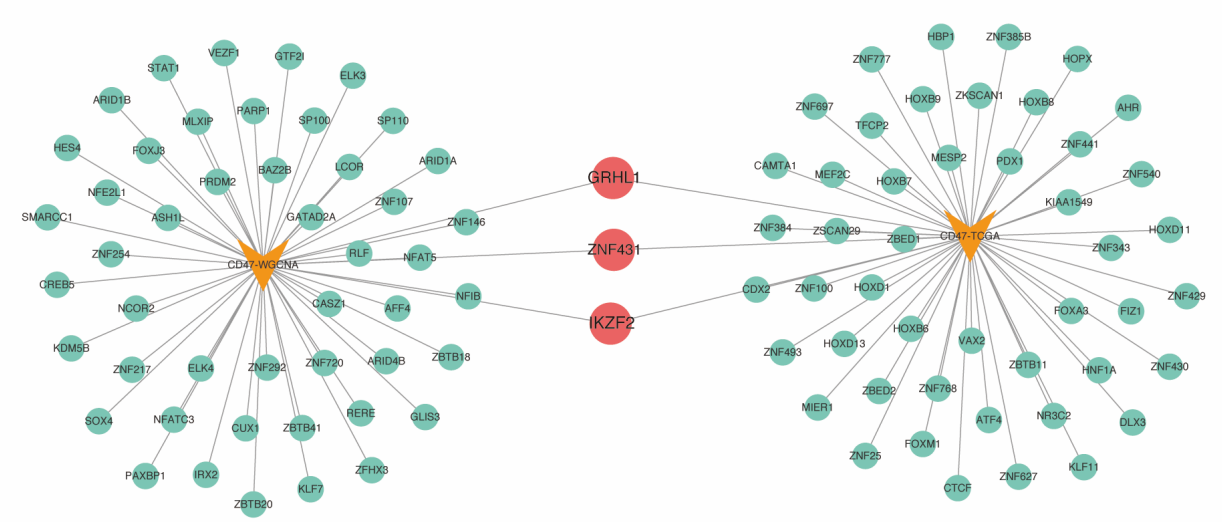

9 performed in our study to intersect with TCGA data in order to identify molecular modulation mechanism. 

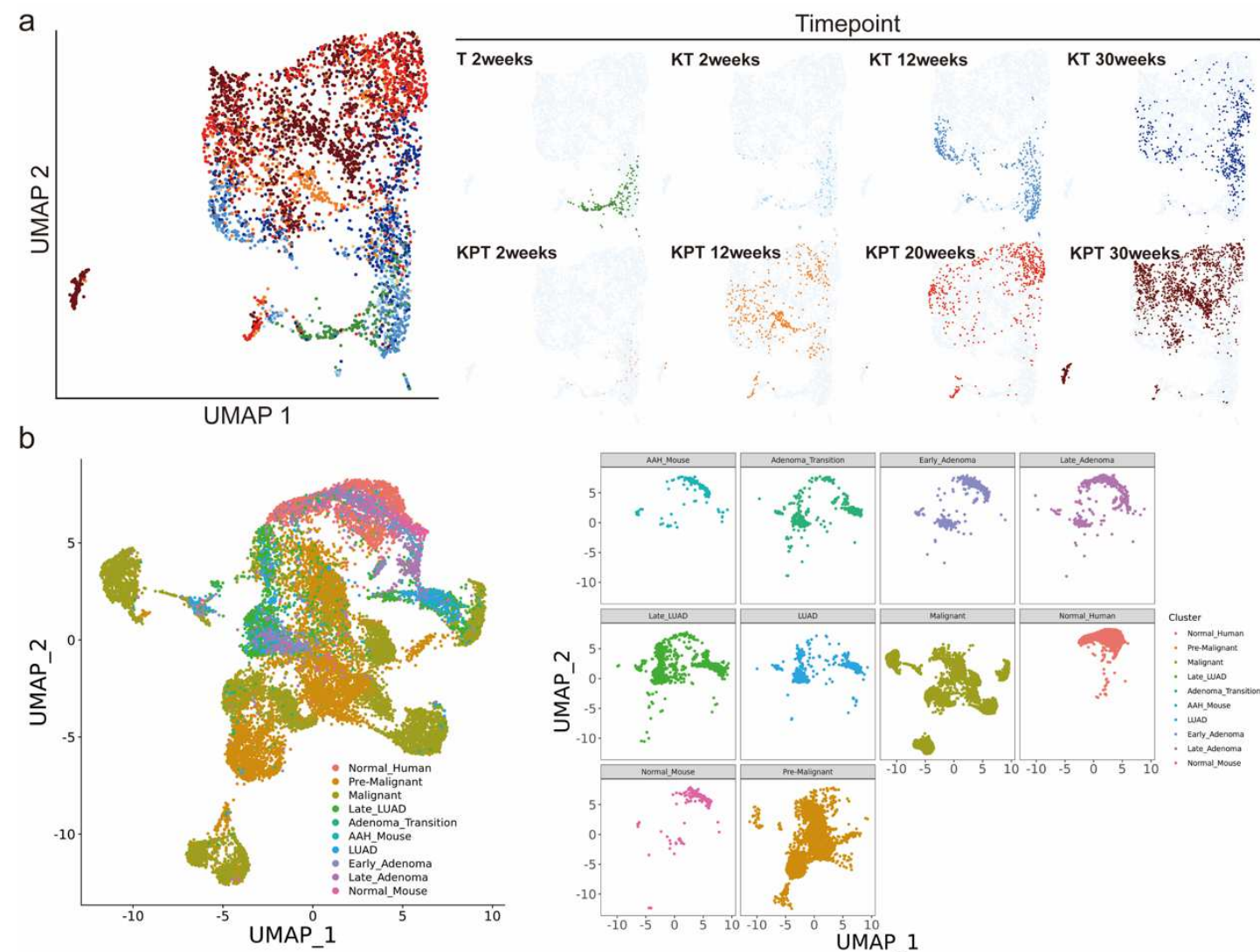

KPT 2weeks

KPT 12weeks

KPT 20weeks Her

KPT 30 weeks $n$ t
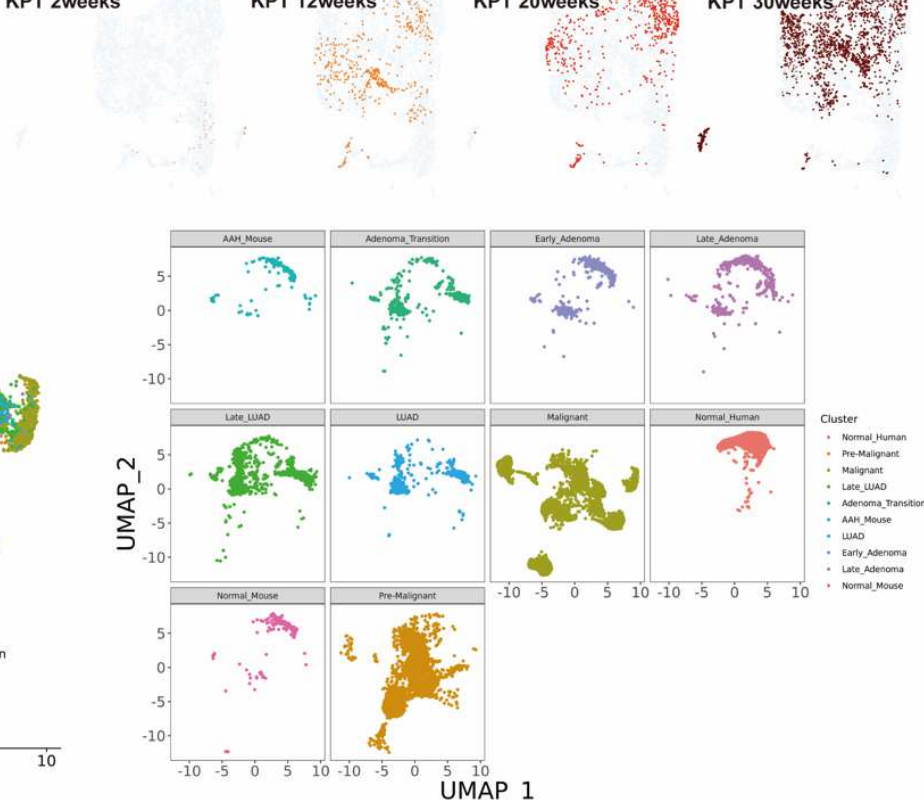

c

KRT18

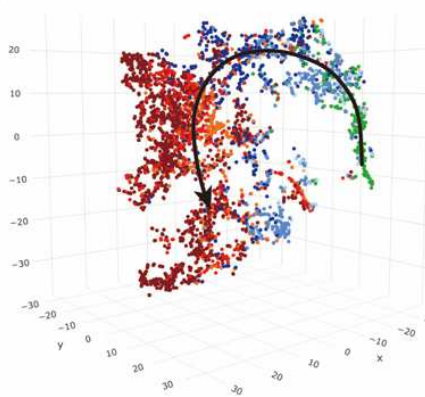

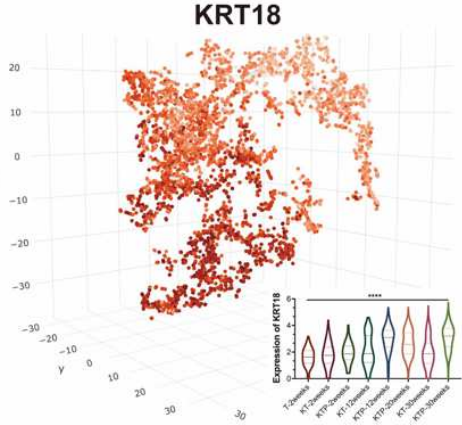

PHLDA2

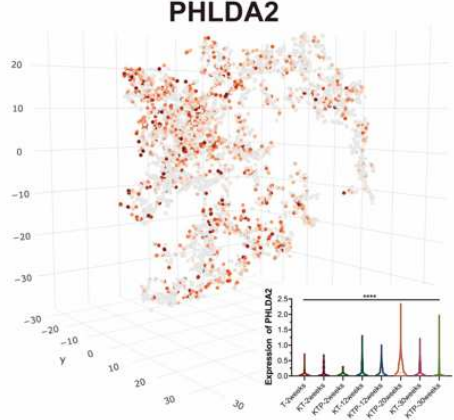
(

\section{Suppl-9. Single-cell data of lung adenocarcinoma derived from mouse model and in} combination with human LUAD single cell data. a, UMAP of lung adenocarcinoma regarding different time point from mouse model. b, Integration of single cell data for tumor cells from our study and mouse model. Both showed similar evolutionary trend upon different stages. c, 3dimensional t-SNE plots of mouse model, and expression of KRT18 and PHLDA2 among different stages. One-way analysis of variance (ANOVA) was used to calculate the significance. 0 1

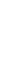
15 
a

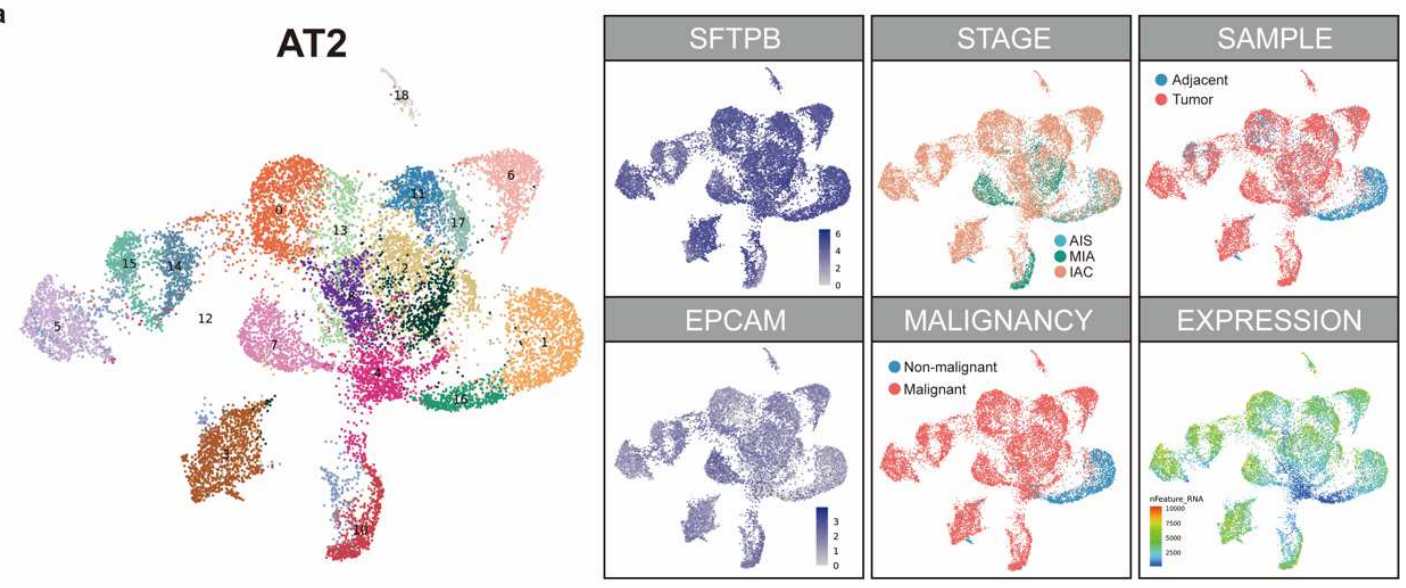

b
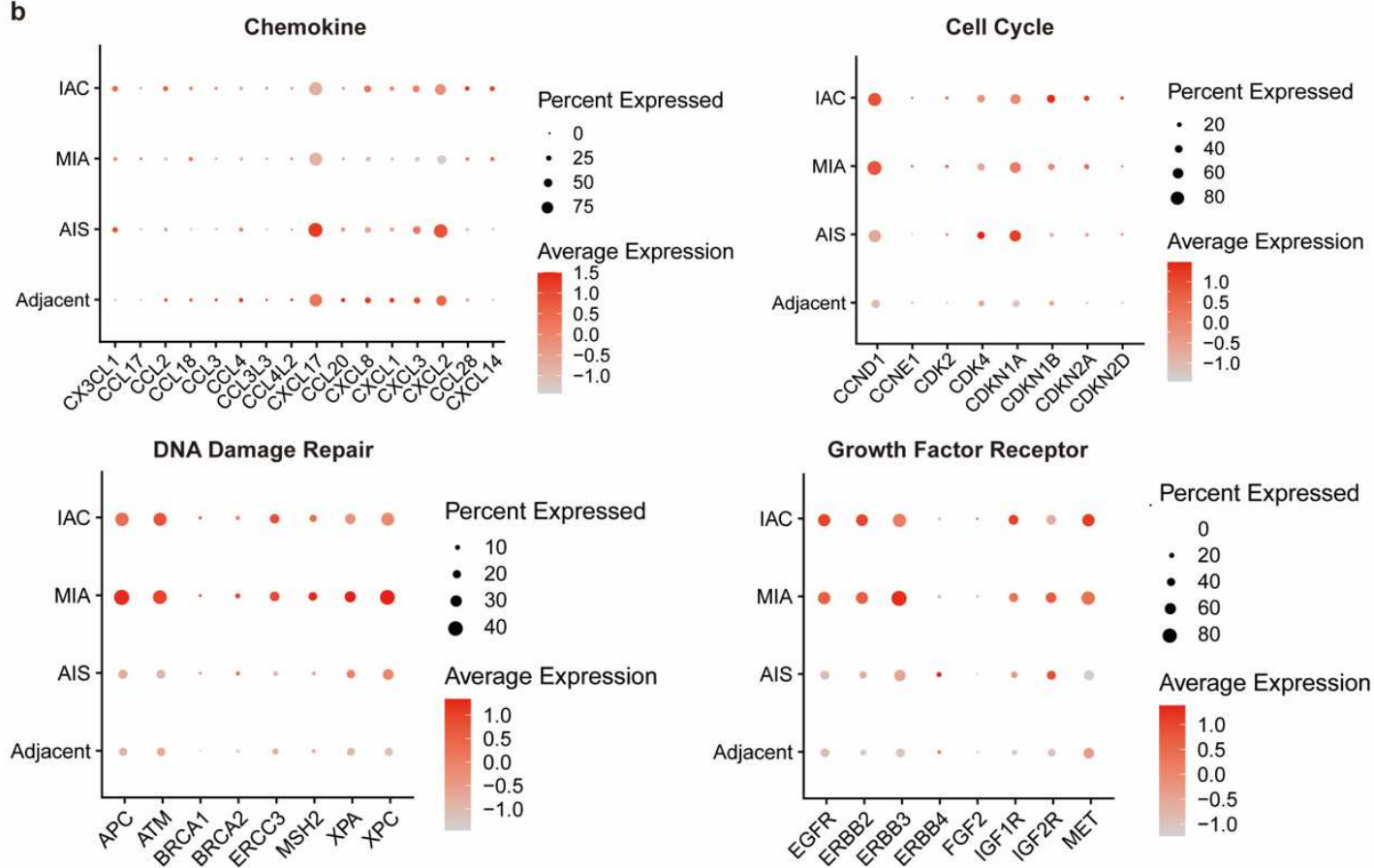

1

Suppl-10. Construction of AT2 cells and expression features. a, Recluster of AT2 cells through t-SNE and overview of distribution. $\mathbf{b}$, Expression of chemokine, cell cycle, DNA damage repair and growth factor receptor related genes among different stages. 


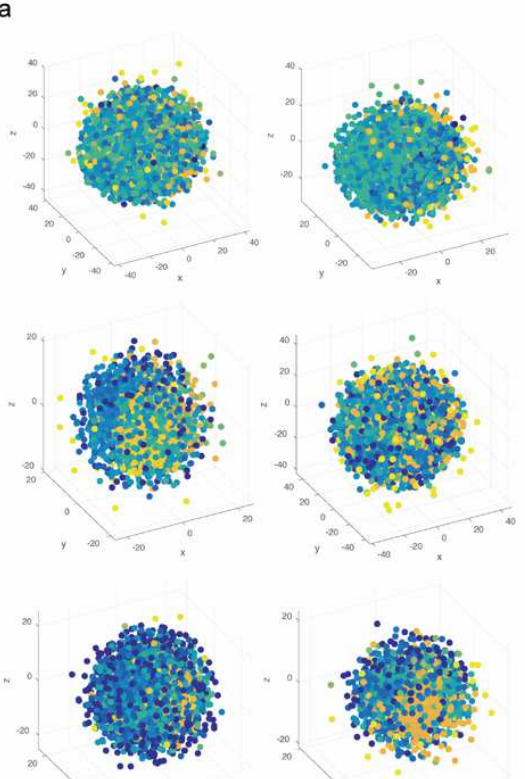

Suppl-11. Reconstruction of spatial cell type distribution through COSmap. a-b,

Representative reconstruction of spatial cell distribution by COSmap and quantified distance for each cell types. $\mathbf{c}$, schematic plot for distribution of different cell types. $\mathbf{d}$, mIHC staining of PANCK, SMA, CD56, CD3, CD8 and GZB in tumor and adjacent tissue.
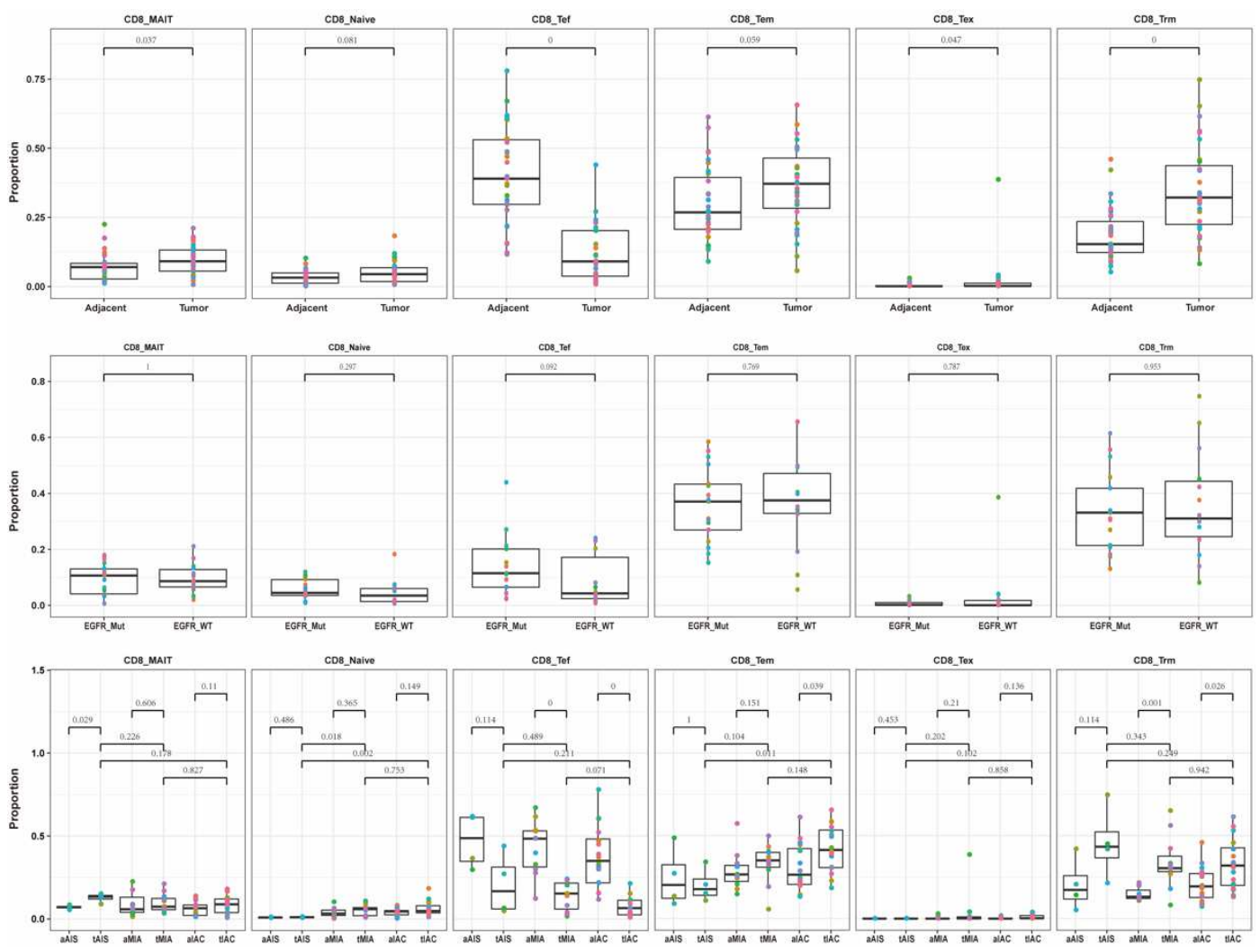

8 Suppl-12. Proportion of CD8 subtypes among different samples, EGFR status and stages.

9 CD8-MAIT, CD8-Naïve, CD8-Tef, CD8-Tem, CD8-Tex and CD8 Trm were measured, and t-test
- AlveolarMac_AIS CD163-DC_AIS CD1A-DC_AIS

CD4_Interferon_high_AIS

CD4_Naive_Als

CD4_Th1-like_AIS

CD4_Th17_AIS

CD4_Trm_AIS

CD4_Undefined3_AIS

CD8_MAIT_AIS

CD8_Tef_AIS

CD8_Tem_AIS

CD8_Trm_AIS

CLEC9A-DC_AIS

Endothelia_AIS

ILC_AIS

Macrophage

Macrophage_M2_AIS

Mast_AIS

Monocyte_AIS

NKT_AIS

Neutrophil_AIS

Plasma_A

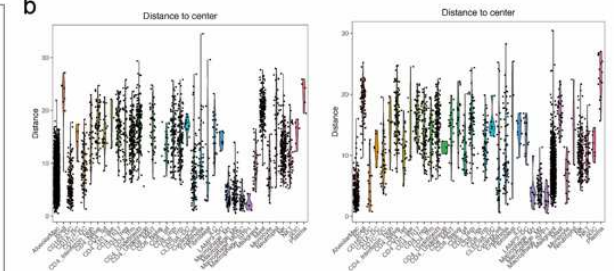

c

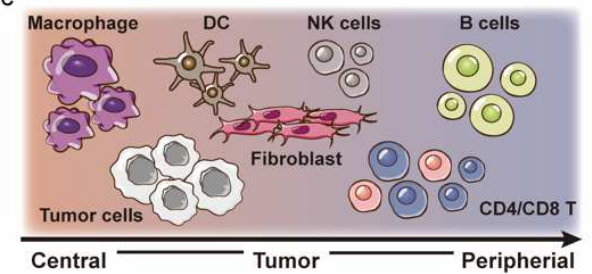

d

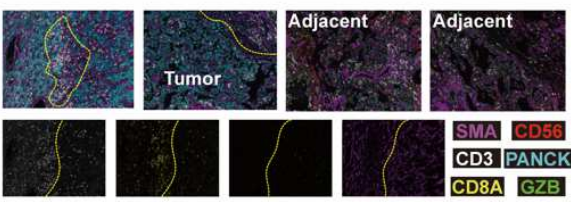



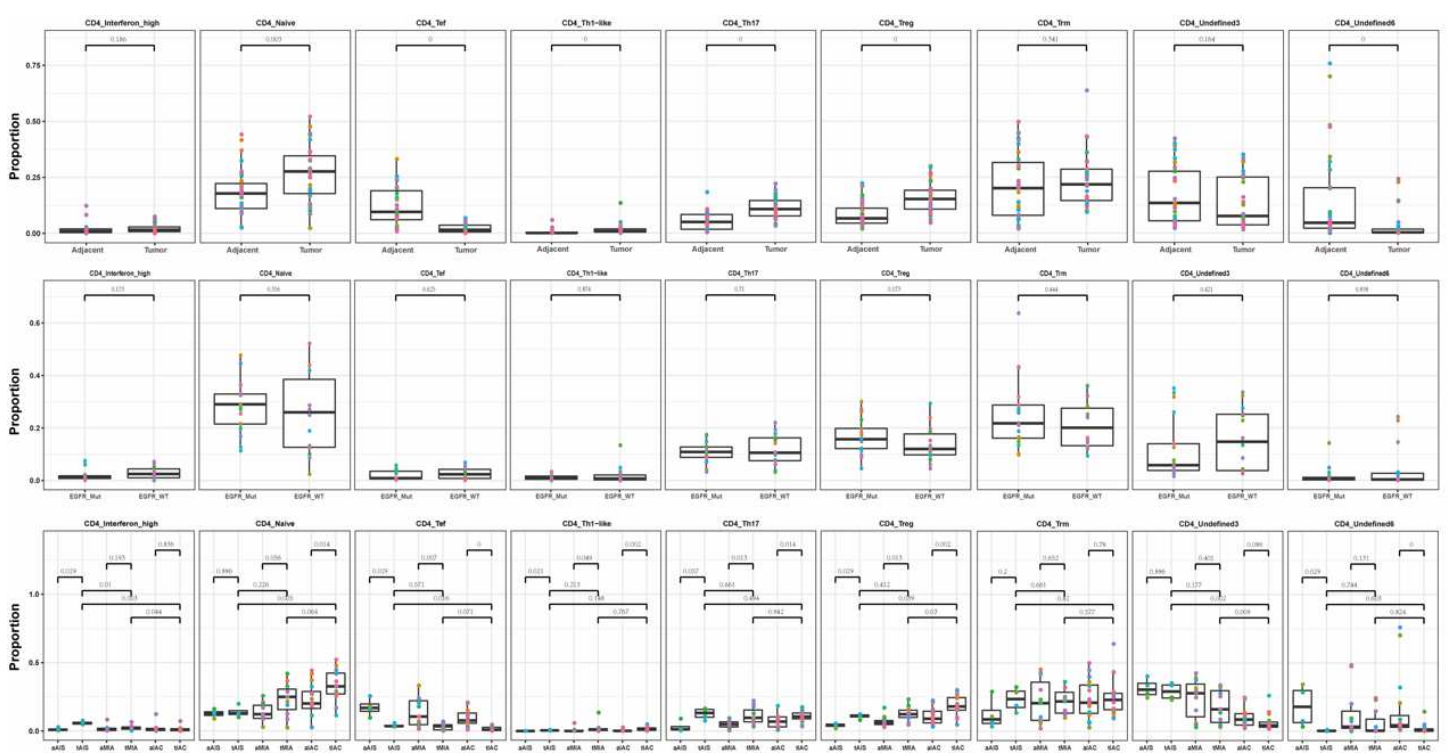

Suppl-13. Correlation of clone size between tumor and adjacent tissue through single-cell

TCR. The left bar in each graph represented tumor specific clonal expansion and lower bar represented adjacent-tissue specific clonal expansion while others represented clonal expansion in both tumor and adjacent tissue.
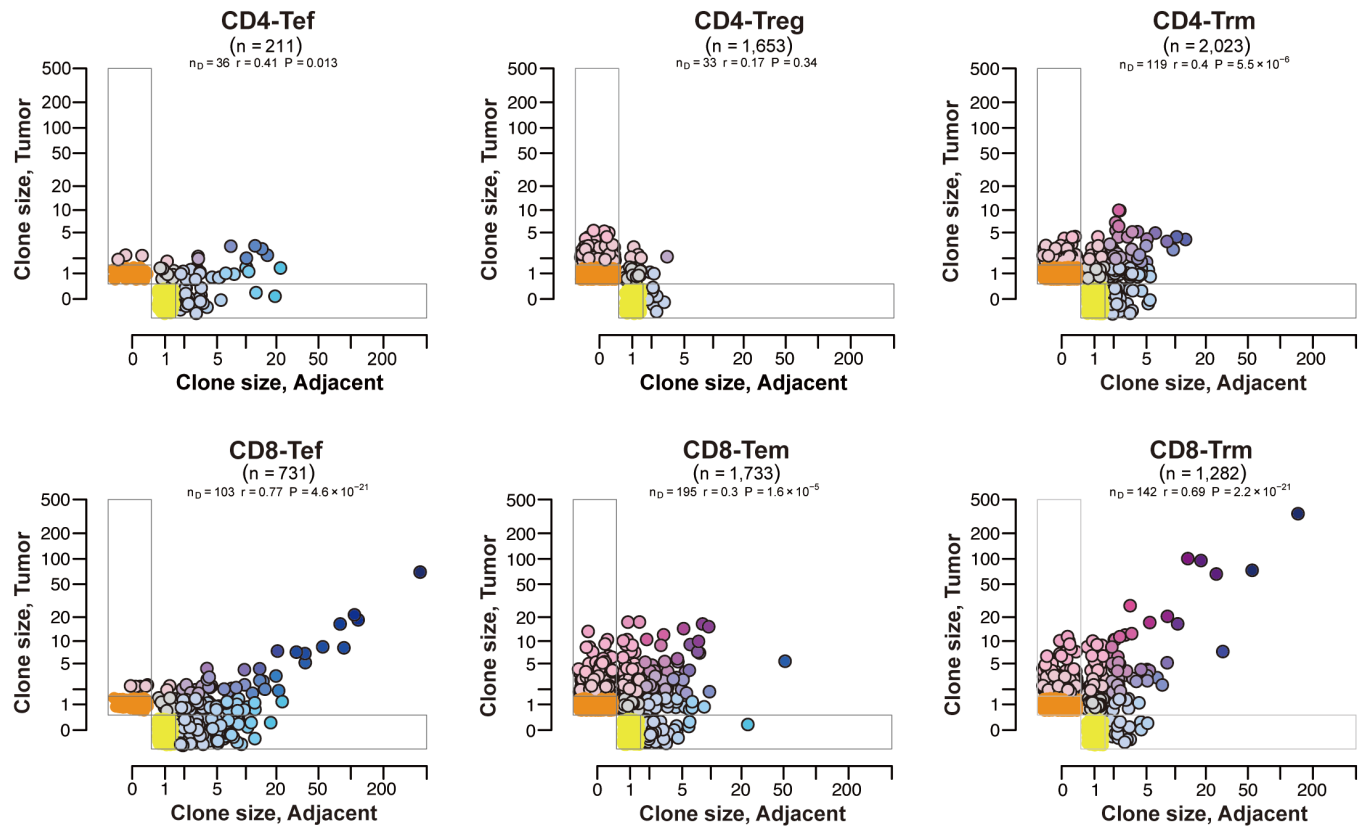

Suppl-14. Proportion of CD4 subtypes among different samples, EGFR status and stages. CD4-interferon high, CD4-Naïve, CD4-Tef, CD4-Th1-like, CD4-Th17, CD4-Treg and CD4 Trm were measured, and t-test between groups were calculated. aAIS, adjacent tissue for AIS. tAIS, tumor tissue for AIS. 

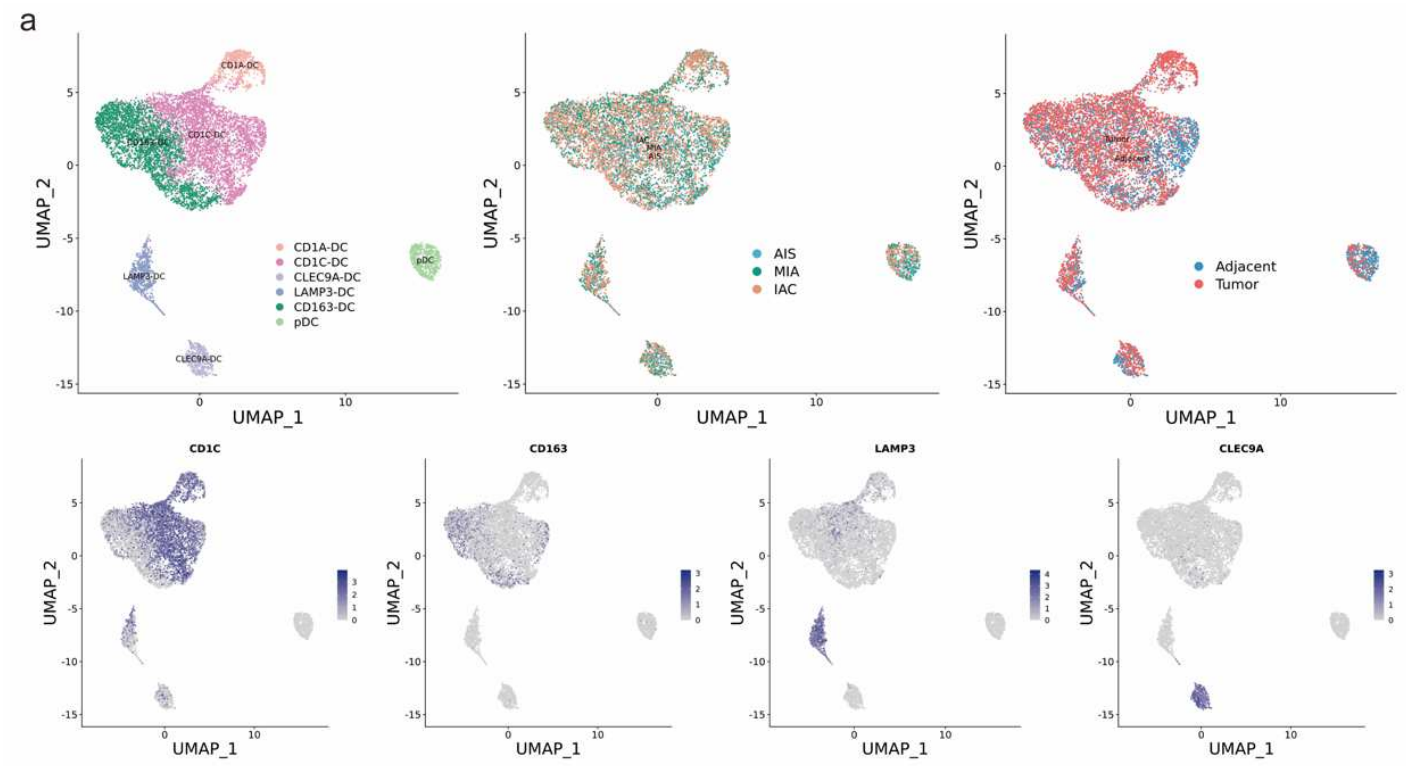

b
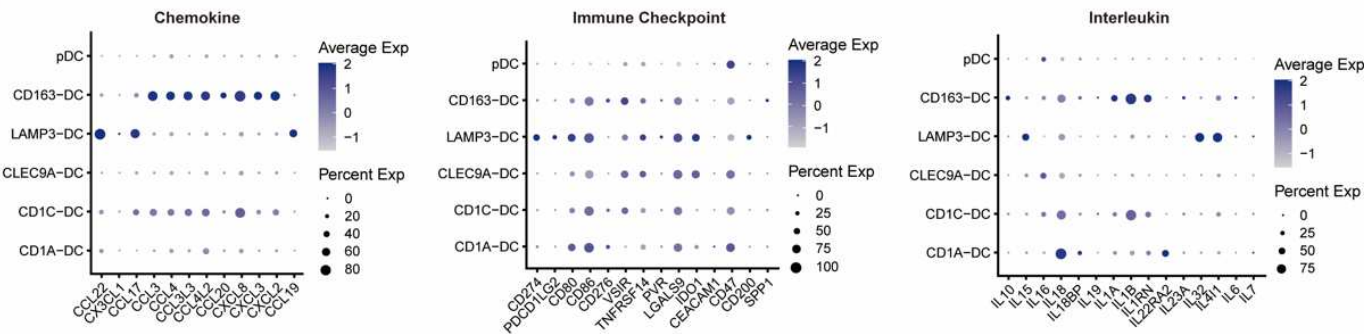

C

d

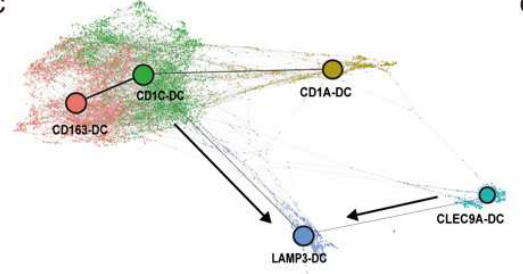

$\stackrel{\mathrm{pDC}}{\mathrm{O}}$

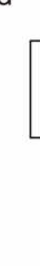

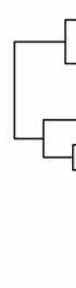

1

2

3

4

Suppl-15. Distribution and function of DC among different stages. a, UMAP of DC along with featured markers. b, Expression of chemokine, immune checkpoint and interleukin related genes among DC subtypes. c, PEGA analysis of different DC subtypes revealed an evolution trajectory of LAMP3-DC. d, Heatmap of functional expression among different DC subtypes. e,

Comparison of LAMP3-DC among different stages and t-test was performed to measure difference between groups. 

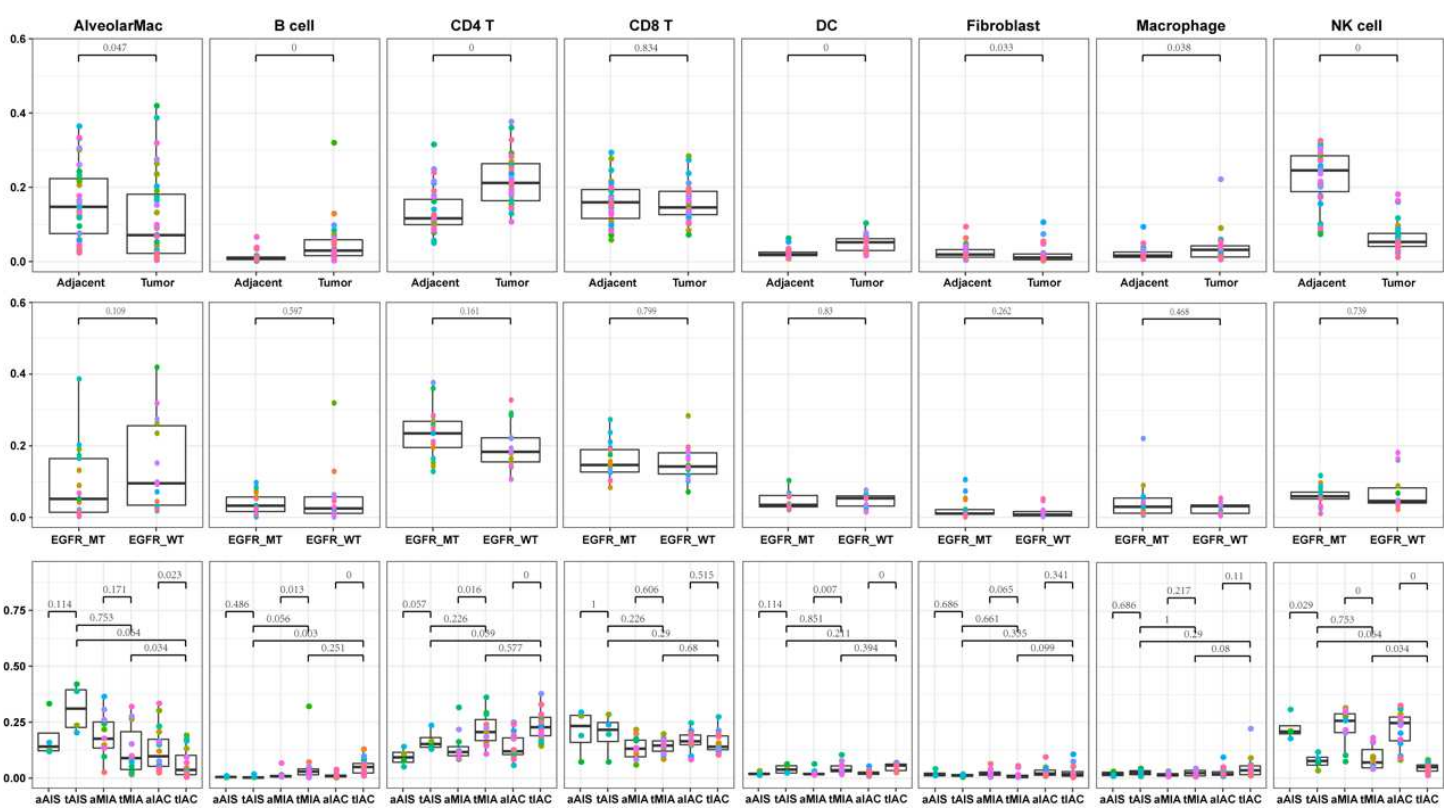

Suppl-16. Proportion of major cell types among different samples, EGFR status and stages.

3 Alveolar macrophage, B cells, CD4 T cells, CD8 T cell, DC, fibroblast cells, macrophages and

4 NK cells were measure, and t-test between groups were calculated. aAIS, adjacent tissue for AIS.

5 tAIS, tumor tissue for AIS. 